\title{
3
}

\section{New Views of Thermonuclear Bursts}

\author{
Tod Strohmayer \\ Laboratory for High Energy Astrophysics \\ NASA's Goddard Space Flight Center, Greenbelt, MD 20771 \\ Lars Bildsten \\ Kavli Institute for Theoretical Physics and Department of Physics \\ University of California, Santa Barbara, CA 93106
}

\subsection{Introduction}

Many accreting neutron stars erupt in spectacular thermonuclear conflagrations every few hours to days. These events, known as Type I X-ray bursts, or simply X-ray bursts, are the subject of our review. Since the last review of Xray burst phenomenology was written (Lewin, van Paradijs \& Taam 1993; hereafter LVT), powerful new X-ray observatories, the Rossi X-ray Timing Explorer (RXTE), the Italian - Dutch BeppoSAX mission, XMM-Newton and Chandra have enabled the discovery of entirely new phenomena associated with thermonuclear burning on neutron stars. Some of these new findings include: (i) the discovery of millisecond $(300-600 \mathrm{~Hz})$ oscillations during bursts, so called "burst oscillations", (ii) a new regime of nuclear burning on neutron stars which manifests itself through the generation of hours long flares about once a decade, now referred to as "superbursts", (iii) discoveries of bursts from low accretion rate neutron stars, and (iv) new evidence for discrete spectral features from bursting neutron stars.

It is perhaps surprising that nuclear physics plays such a prominent role in the phenomenology of an accreting neutron star, as the gravitational energy released per accreted baryon (of mass $m_{p}$ ) is $G M m_{p} / R \approx 200 \mathrm{MeV}$ is so much larger than the nuclear energy released by fusion $(\approx 5 \mathrm{MeV}$ when a solar mix goes to heavy elements). Indeed, if the accreted fuel was burned at the rate of accretion, any evidence of nuclear physics would be swamped by the light from released gravitational energy. The only way the nuclear energy can be seen is when the fuel is stored for a long period and then burns rapidly (as in Type I bursts and Superbursts).

The advances in millisecond timing were enabled by RXTE's combination of large X-ray collecting area, sub-millisecond time resolution and a state-of-the-art data system and telemetry capacity. RXTE's instruments are also unfettered; they can look where the action is for longer, more often, and more quickly than any previous X-ray observatory. The Wide Field Cameras (WFC) on BeppoSAX (Jager et al. 1997), in combination with the All Sky Monitor (ASM) onboard RXTE (Levine et al. 1996), have provided an unprecedented, long term view of the X-ray sky that opened up new discovery space for rare events. Their capabilities led to the discovery of superbursts as a new manifestation of thermonuclear burning on neutron stars, as well as the detection of rare bursts from neutron stars accreting at very low rates. Indeed, WFC observations alone have led to the discovery of about 20 new bursters (see in 't Zand 2001). 
In addition to these new results, recent discoveries have provided answers to some long standing questions concerning the sources of thermonuclear bursts, the neutron star low mass X-ray binaries (LMXB). These include; (i) the discovery of accreting millisecond pulsars in three LMXB systems, confirming that neutron stars are spunup to millisecond periods by accretion, (ii) the discovery of sub-millisecond variability from many neutron star LMXBs (see the review by van der Klis in Chapter 2) and (iii) detections of several bursting neutron star transients (e.g. Aql X-1, Cen X-4) in quiescence with the increased sensitivity afforded by Chandra and XMM/Newton. These data have provided important new information on the thermal state of neutron stars which strongly influences their bursting behavior (see Chakrabarty \& Psaltis in Chapter 1).

Our review focuses on the new phenomena and insights they provide about neutron stars and thermonuclear burning on them. These discoveries have stimulated much new theoretical thinking. In particular, the discovery of millisecond oscillations during bursts has refocused attention on the need to understand the multidimensional nature of thermonuclear flame propagation, that is, how burning is initiated and how it spreads around the neutron star. An understanding of flame propagation, in turn, requires an understanding of how thermonuclear burning influences the dynamics of the accreted layers. To understand superbursts, theorists have been forced to think about what happens at much greater depths in the accreted layers of neutron stars, and about the detailed composition of the "ashes" of $\mathrm{H} / \mathrm{He}$ burning. Finally, observations of bursts from sources with very low mass accretion rates are forcing theorists to confront some physical processes, for example, element diffusion, which have not been considered in earlier work.

Space constraints do not allow us to present an exhaustive review of all X-ray burst phenomenology and theory. For example, we will not describe in detail the Type II (accretion instability) burst phenomena now observed from two sources; the famous Rapid Burster (see for example, LVT); and the Bursting Pulsar (GRO J174428, see Kouveliotou et al. 1996; Giles et al. 1996). Although new observations of Type II bursters have been obtained since the last reviews were written, including the discovery of GRO J1744-28, only the 2nd known Type II burster, they have generally not provided new qualitative insights on the accretion instability. Some of the more recent observations of these sources, and our current understanding of them, are described elsewhere in this volume (see the contributions by Lewin \& Verbunt; Chakrabarty \& Psaltis; and van der Klis).

Despite its energetic disadvantage, we are now confident that sudden nuclear energy release powers bursts and, as we emphasize here, fleshing out the details of the thermonuclear flash model in the context of the observations is probing both neutron star structure and fundamental physics. We try not to segregate our discussions into separate observational and theoretical pieces, rather, we integrate the theory and observations as they relate to particular phenomena as much as possible. In that sense, this is an incomplete review of the state of the theoretical research in this field.

We begin in $\S 2$ with a brief theoretical introduction to the relevant physics of thermonuclear burning on neutron stars and how it accounts for the gross properties of bursts. We then briefly review the observational characteristics of bursts and sources. This will lay out the fundamentals and topics important for our discussions 
of the new results. Here we also include some discussion on new results from burst spectroscopy. We refer the reader to Bildsten (1998) for relevant theoretical details.

We then move to the new discoveries. In $\S 4$ we introduce millisecond variability during bursts ("burst oscillations"), and theoretical implications motivated by the observations. In $\S 5$ we describe the new observations of superbursts and their theoretical implications. We close in $\S 6$ with a summary and some comments on the future prospects of burst research.

\subsection{The physics of hydrogen-helium burning}

At the core of the observed phenomena is the "thin shell" instability discovered theoretically by Schwarzschild \& Härm (1965) in the helium shell residing above the carbon/oxygen core during the asymptotic giant branch phase of stellar evolution. The driver of this instability is a nuclear energy generation rate that is more temperature sensitive than radiative cooling and is confined to a thin shell. Hansen \& Van Horn (1975) showed that burning of the accumulated hydrogen and helium on a neutron star also occurs in radially thin shells that were susceptible to the same instability. Soon thereafter, type I X-ray bursts from LMXBs were independently discovered by Grindlay et al. (1976) and Belian, Conner \& Evans (1976), and were quickly associated (Woosley \& Taam 1976; Maraschi \& Cavaliere 1977; Joss 1977, 1978; Lamb \& Lamb 1978) with Hansen \& Van Horn's (1975) instability. For a brief historical overview see LVT (1993).

The successful association of thermonuclear instabilities with X-ray bursts made a nice picture of a recurrent cycle that consists of fuel accumulation for several hours to days followed by a thermonuclear runaway that burns the fuel in $\sim 10-100$ seconds. It also secured the identification of the accreting objects as neutron stars (NS). The mass donors - the ultimate source of the thermonuclear fuel - are typically old, Population II objects or in some cases, degenerate helium or perhaps carbon/oxygen white dwarfs (Rappaport, Joss \& Webbink 1982). The accreted composition is important, as the nuclear ashes and burst properties depend on the accreted mix of light elements. Unfortunately, in most cases, we have little information on the composition of the accreted fuel. Of the approximately 160 known LMXB's about 70 are observed to produce bursts (see Liu, van Paradijs \& van den Heuvel 2001; Chakrabarty \& Psaltis in Chapter 1).

A fundamental physical reason for studying the neutron star example of a thin shell instability is that the timescales are observationally accessible. Hundreds of bursts have been seen from some neutron stars, and study of the burst dependence on accretion rate can be undertaken. Such an exercise is rare to impossible in the other astrophysical site where the instability is observable: the explosion of hydrogen on an accreting white dwarf as a classical nova. For a more detailed theoretical discussion see Bildsten (1998).

\subsubsection{Nuclear burning during accumulation and ignition}

After the accreted hydrogen and helium has become part of the NS, it undergoes hydrostatic compression as new material is piled on. The fresh fuel reaches ignition densities and temperatures within a few hours to days. The resulting compression rate depends on the accretion rate per unit area, $\dot{m} \equiv \dot{M} / A_{a c c}$, where $A_{a c c}$ 
is the area covered by fresh material. We will sometimes quote numbers for both $\dot{m}$ and $\dot{M}$. When we give $\dot{m}$, we have assumed $A_{a c c}=4 \pi R^{2} \approx 1.2 \times 10^{13} \mathrm{~cm}^{2}$. The time it takes for heat transport to cool the deep envelope (what we call the thermal time) is only ten seconds at the ignition location, where the pressure is $P \approx 10^{22}-10^{23} \mathrm{erg} \mathrm{cm}^{-3}$. This is so much shorter than the time to accumulate the material (hours to days) that the compression is far from adiabatic.

The temperature exceeds $10^{7} \mathrm{~K}$ in most of the accumulating atmosphere, so that hydrogen burns via the CNO cycle and the proton - proton (pp) cycle can be neglected. At high temperatures $\left(T>8 \times 10^{7} \mathrm{~K}\right)$, the timescale for proton captures becomes shorter than the subsequent $\beta$ decay lifetimes, even for the slowest ${ }^{14} \mathrm{~N}(\mathrm{p}, \gamma){ }^{15} \mathrm{O}$ reaction. The hydrogen then burns in the "hot" CNO cycle of Fowler \& Hoyle (1965)

$$
{ }^{12} \mathrm{C}(p, \gamma){ }^{13} \mathrm{~N}(p, \gamma){ }^{14} \mathrm{O}\left(\beta^{+}\right){ }^{14} \mathrm{~N}(p, \gamma){ }^{15} \mathrm{O}\left(\beta^{+}\right){ }^{15} \mathrm{~N}(p, \alpha){ }^{12} \mathrm{C},
$$

and is limited to $5.8 \times 10^{15} Z_{\mathrm{CNO}}$ ergs g ${ }^{-1} \mathrm{~s}^{-1}$, where $Z_{\mathrm{CNO}}$ is the mass fraction of CNO. The hydrogen burns this way in the accumulating phase when,

$$
\dot{m}>900 \mathrm{~g} \mathrm{~cm}^{-2} \mathrm{~s}^{-1}\left(Z_{C N O} / 0.01\right)^{1 / 2},
$$

and is thermally stable. The amount of time it takes to burn the hydrogen is $\approx$ $\left(10^{3} / Z_{\mathrm{CNO}}\right) \mathrm{s}$, or about one day for solar metallicities. This time is even longer if the donor star has a low metal content or if there is substantial spallation of the incident CNO elements as discussed by Bildsten, Salpeter and Wasserman (1992). For lower $\dot{m}$ 's, the hydrogen burning is thermally unstable and can trigger Type I bursts.

The slow hydrogen burning during accumulation allows for a unique burning regime at high $\dot{m}$ 's. This simultaneous $\mathrm{H} / \mathrm{He}$ burning occurs when

$$
\dot{m}>2 \times 10^{3} \mathrm{~g} \mathrm{~cm}^{-2} \mathrm{~s}^{-1}\left(Z_{C N O} / 0.01\right)^{13 / 18},
$$

(Bildsten 1998; Cumming \& Bildsten 2000), as at these high rates the fluid is compressed to helium ignition conditions before the hydrogen is completely burned (Lamb \& Lamb 1978; Taam \& Picklum 1978). The strong temperature dependence of the helium burning rate (and lack of any weak interactions) leads to a thin-shell instability for temperatures $T<5 \times 10^{8} \mathrm{~K}$ and causes the burst. The critical condition of thin burning shells $(h \ll R)$ is true before burning and remains so during the flash. Stable burning sets in at higher $\dot{m}$ 's (comparable to the Eddington limit; Paczynski 1983; Bildsten 1998, Narayan \& Heyl 2002) when the helium burning temperature sensitivity finally becomes weaker than the cooling rate's sensitivity (Ayasli \& Joss 1982; Taam, Woosley \& Lamb 1996). This is consistent with the absence of bursts from high-field X-ray pulsars, which channel the accretion flow onto a small-area polar cap, and thus achieve a high local (and stabilizing) $\dot{m}$ (Joss \& Li 1980; Bildsten \& Brown 1997).

For solar metallicities, there is a narrow window of $\dot{m}$ 's where the hydrogen is completely burned before the helium ignites. In this case, a pure helium shell accumulates underneath the hydrogen-burning shell until conditions are reached for ignition of the pure helium layer. The recurrence times of these bursts must be longer than the time to burn all of the hydrogen, so pure helium flashes should have recurrence times in excess of a day and $\alpha \approx 200$ ( $\alpha$ is the ratio of the time-averaged persistent to 
burst luminosity). To summarize, in order of increasing $\dot{m}$, the regimes of unstable burning we expect from NSs accreting at sub-Eddington rates $\left(\dot{m}<10^{5} \mathrm{~g} \mathrm{~cm}^{-2} \mathrm{~s}^{-1}\right)$ are (Fujimoto, Hanawa \& Miyaji 1981; Fushiki \& Lamb 1987; Cumming \& Bildsten 2000):

(1) Mixed hydrogen and helium burning triggered by thermally unstable hydrogen ignition for $\dot{m}<900 \mathrm{~g} \mathrm{~cm}^{-2} \mathrm{~s}^{-1}\left(\dot{M}<2 \times 10^{-10} M_{\odot} \mathrm{yr}^{-1}\right)$.

(2) Pure helium shell ignition for $900 \mathrm{~g} \mathrm{~cm}^{-2} \mathrm{~s}^{-1}<\dot{m}<2 \times 10^{3} \mathrm{~g} \mathrm{~cm}^{-2} \mathrm{~s}^{-1}$ following completion of hydrogen burning.

(3) Mixed hydrogen and helium burning triggered by thermally unstable helium ignition for $\dot{m}>2 \times 10^{3} \mathrm{~g} \mathrm{~cm}^{-2} \mathrm{~s}^{-1}\left(\dot{M}>4.4 \times 10^{-10} M_{\odot} \mathrm{yr}^{-1}\right)$.

The transition $\dot{m}$ 's are for $Z_{C N O} \approx 0.01$. Reducing $Z_{C N O}$ lowers the transition accretion rates and substantially narrows the $\dot{m}$ range for pure helium ignition. Another effect critical to the burning is the amount of heat flux coming through the burning layer from deeper parts of the NS ocean (Ayasli \& Joss 1982, Fushiki \& Lamb 1987). The current theoretical estimates (Brown, Bildsten \& Rutledge 1998; Brown 2000; Colpi et al. 2001) are that between 10 and $100 \%$ of the heat released via pycnonuclear reactions in the deep crust (Haensel \& Zdunik 1990) emerges from the surface, proving most important to the burst properties of pure helium accretors such as 4U 1820-30 (Bildsten 1995, Strohmayer \& Brown 2002).

\subsubsection{Burning during the bursts: the rp-process}

We now briefly discuss what happens as the thermal instability develops into a burst and what observational differences are to be expected between a pure helium ignition and a mixed hydrogen/helium ignition. The flash occurs at fixed pressure, and the increasing temperature eventually allows the radiation pressure to dominate. For a typical ignition column of $2 \times 10^{8} \mathrm{~g} \mathrm{~cm}^{-2}$, the pressure is $P=$ $g y \approx 4 \times 10^{22} \mathrm{ergs} \mathrm{cm}^{-3}$, so $a T_{\max }^{4} / 3 \approx P$ gives $T_{\max } \approx 2 \times 10^{9} \mathrm{~K}$. For pure helium flashes, the fuel burns rapidly (since there are no slow weak interactions) and the local Eddington limit is often exceeded. These conditions lead to photospheric radius expansion (PRE) bursts with durations, set mostly by the time it takes the heat to escape, of order $5-10$ seconds.

When hydrogen and helium are both present, the temperatures reached during the thermal instability can easily produce elements far beyond the iron group (Hanawa et al. 1983; Wallace \& Woosley 1984; Hanawa \& Fujimoto 1984; Koike et al. 1999; Schatz et al. 1999, 2001) via the rapid-proton (rp) process of Wallace \& Woosley (1981). This burning starts after the triggering helium flash heats the gas to high enough temperatures to allow the "breakout" reactions ${ }^{15} \mathrm{O}(\alpha, \gamma){ }^{19} \mathrm{Ne}$ and ${ }^{18} \mathrm{Ne}(\alpha, p){ }^{21} \mathrm{Na}\left({ }^{18} \mathrm{Ne}\right.$ is made in the chain ${ }^{14} \mathrm{O}(\alpha, p){ }^{17} \mathrm{~F}(p, \gamma){ }^{18} \mathrm{Ne}\left(\beta^{+}\right){ }^{18} \mathrm{~F}(p, \alpha){ }^{15} \mathrm{O}$ triggered by the high temperatures) to proceed faster than the $\beta$ decays. This takes these catalysts out of the CNO cycle loop, where they subsequently burn hydrogen via the rp process: a series of successive proton captures and $\beta$ decays.

Figure 3.1 shows the dominant path of the nuclei as they move up the protonrich side of the valley of stability (much like the r-process which occurs by neutron captures on the neutron rich side) more or less limited by the $\beta$-decay rates. Theoretical work shows that the end-point of this time-dependent burning is far beyond 


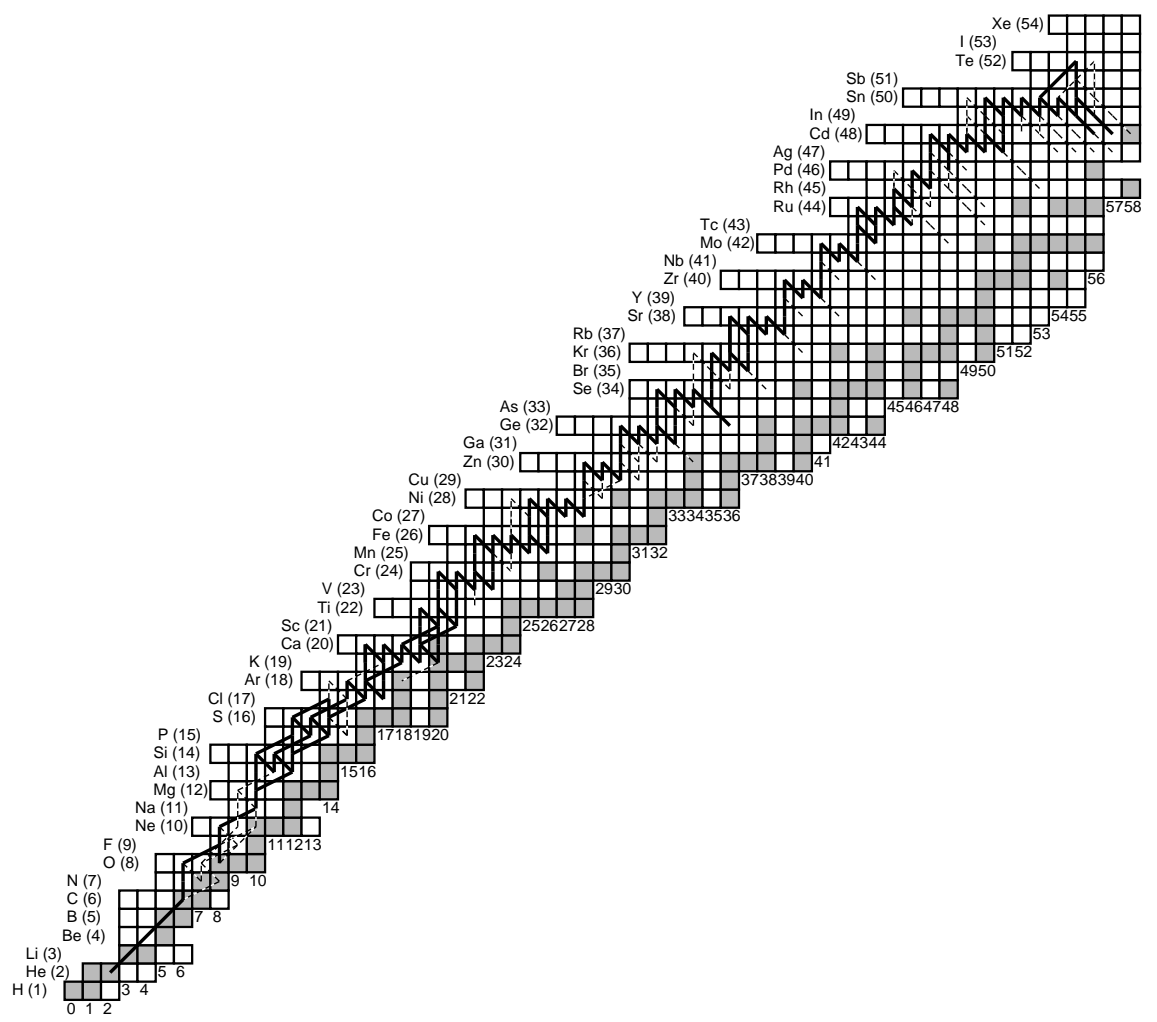

Fig. 3.1. Schematic showing the dominant pathways of the nuclear reaction flows during the rp process. Elements far beyond ${ }^{56} \mathrm{Fe}$ can easily be reached. Filled squares denote stable nuclides (after Schatz et al. 2001).

iron (Hanawa \& Fujimoto 1984, Schatz et al. 1999, 2001, Koike et al. 1999) and is set by either the complete burning of the hydrogen or reaching the closed SnSbTe cycle found by Schatz et al. (2001). When hydrogen is exhausted prior to reaching the $\mathrm{SnSbTe}$ cycle, a rough estimate of the rp-process end-point is made by merely accounting for the ratios of seed nuclei to hydrogen. This produces a large range of heavy nuclei (Schatz et al. 1999, 2001). Schatz et al. (1999) also showed an additional important point of nucleosynthesis, which is that the hydrogen burns out before the helium is completely burned. Thus, the carbon made during late helium burning remains as carbon since there are no protons available for it to capture. As we will show in Section 5, this remaining carbon is the apparent fuel for the recently discovered superbursts. Observationally, the long series of $\beta$ decays during the rpprocess releases energy for at least 150 seconds after the burst has started. We thus expect a mixed hydrogen/helium burst to last much longer than a pure helium burst.

\subsubsection{Mixed $\mathrm{H} / \mathrm{He}$ bursts from GS 1826-238}

We discuss shortly that most bursters do not precisely match the theory presented above in a simple way, and initial indications are that this could be due 


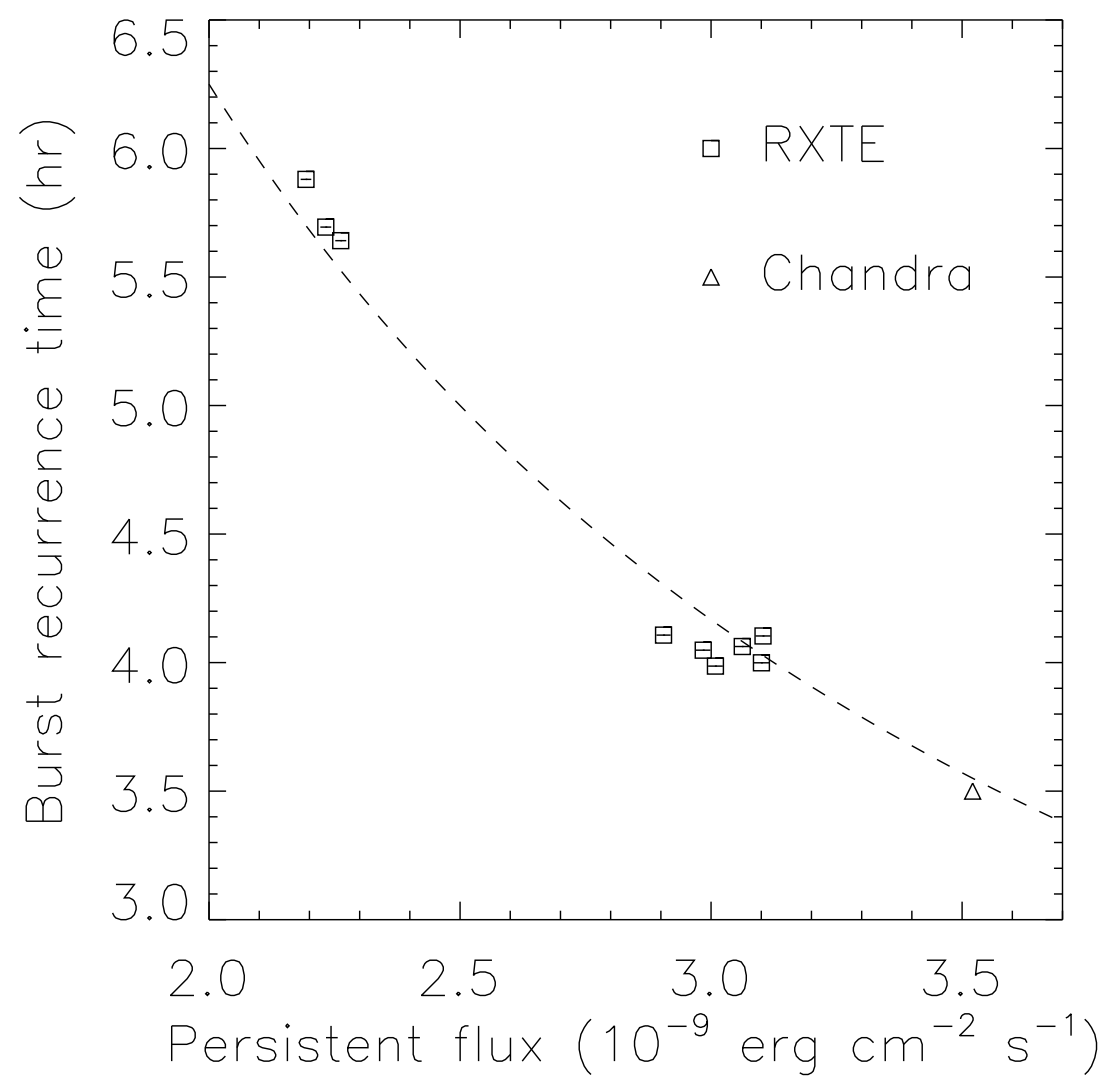

Fig. 3.2. Variation of the burst recurrence time for GS $1826-238$ as a function of the persistent flux from RXTE measurements between 1997-2000 (squares) and a more recent Chandra/RXTE measurement on 2002 July 29. Horizontal error bars indicate the $1 \sigma$ errors. The dashed line is the trend expected if the burst recurrence time is $\propto \dot{M}^{-1}$ (after Galloway et al. 2003).

to variations in the area on which new fuel accretes (Bildsten 2000). However, there are times when bursters behave in a limit cycle manner, with bursts occurring periodically (e.g. Robinson \& Young 1997) as $\dot{m}$ stays at a fixed value and we discuss one beautiful example here: the Type I burster GS 1826-238. Ubertini et al. (1999) found 70 bursts over a 2.5 year monitoring baseline with the BeppoSAX/WFC. The quasi-periodic recurrence time was $5.76 \pm 0.62$ hours. Cocchi et al.'s (2000) later analysis within observing seasons found that in 1997 and 1998 the recurrence times were even more clock-like, $5.92 \pm 0.07$ hours and $5.58 \pm 0.09$ hours. The persistent flux during this bursting period was $F_{x} \approx 2 \times 10^{-9} \mathrm{erg} \mathrm{cm}^{-2} \mathrm{~s}^{-1}$ (Ubertini et al. 1999, in 't Zand et al. 1999, Kong et al. 2000). Since then, the persistent flux has risen, and remarkably, the burst recurrence time has shortened $\propto \dot{M}^{-1}$ (see Figure 3.2, Galloway et al. 2003), implying that the accumulated mass is the same as $\dot{M}$ changes. 


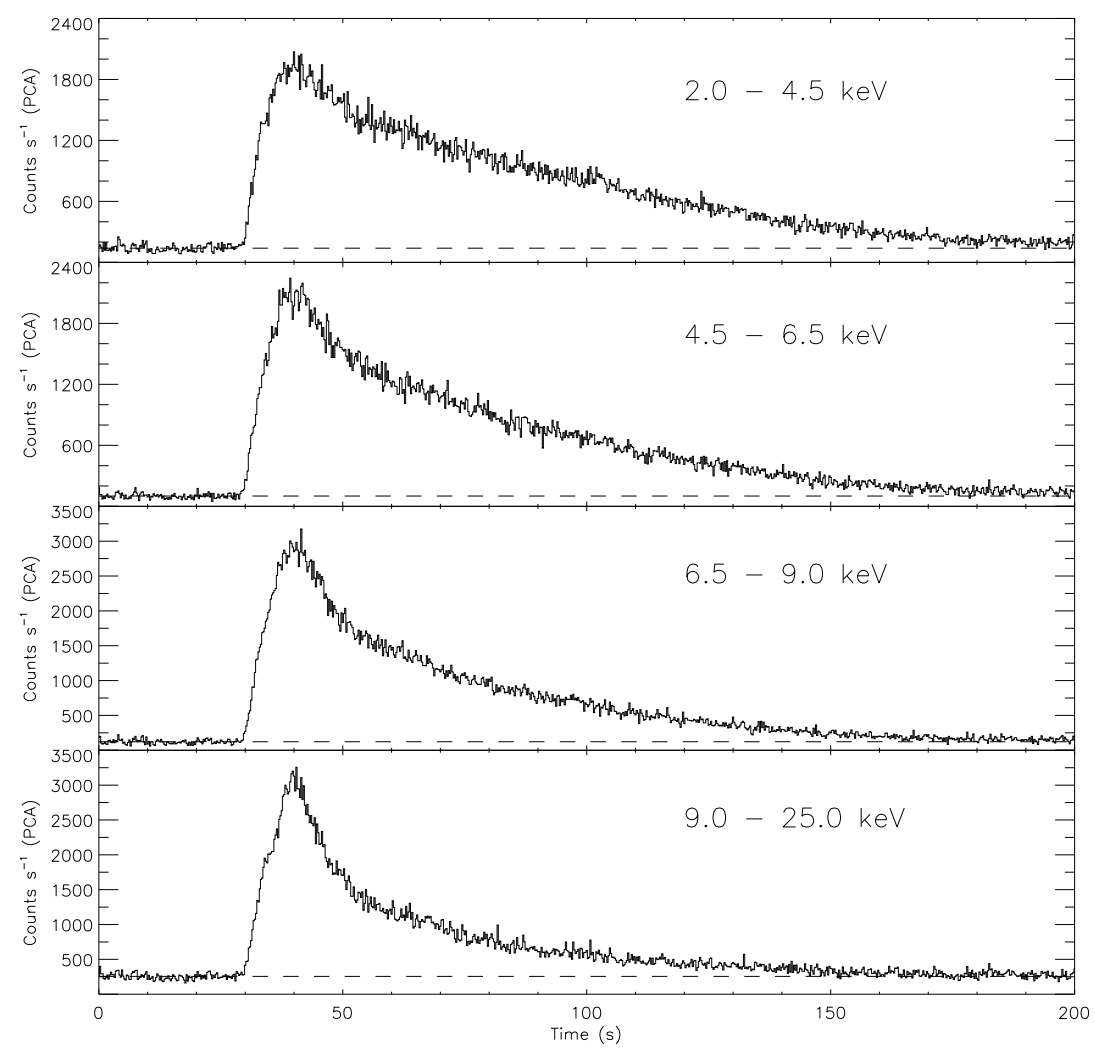

Fig. 3.3. An X-ray burst from GS 1826-238 seen with the RXTE/PCA. The burst is shown in four different energy bands. The long duration is indicative of the delayed energy release from the rapid proton ( $\mathrm{rp}$ ) process. The dashed line marks the preburst flux level (see also Kong et al. 2000).

From the observed persistent flux and the measured ratio of $R_{a p p} / d$ (about $10 \mathrm{~km}$ at $10 \mathrm{kpc}$; Galloway et al. 2003) the local accretion rate is $\dot{m} \approx 10^{4} \mathrm{~g} \mathrm{~cm}^{-2} \mathrm{~s}^{-1}$ when $F_{x} \approx 2 \times 10^{-9} \mathrm{erg} \mathrm{cm}^{-2} \mathrm{~s}^{-1}$. This is very safely in the mixed $\mathrm{H} / \mathrm{He}$ burning regime. The estimated $\dot{m}$ gives an accumulated column on the NS prior to the burst of $2 \times 10^{8} \mathrm{~g} \mathrm{~cm}^{-2}$, just what is expected from theory (see Table 2 of Cumming \& Bildsten 2000). These periodic bursts allow for a very secure measurement of $\alpha \approx 40$ (Galloway et al. 2003), implying a nuclear energy release of $5 \mathrm{MeV}$ per accreted nucleon for a $1.4 M_{\odot}, 10 \mathrm{~km}$ NS. Energy releases this large can only come about via hydrogen burning and the long (>100 s) duration of the bursts are consistent with the expected energy release from the rp-process. Figure 3.3 shows the time profile of a burst seen with the Proportional Counter Array (PCA) on RXTE (see also Kong et al. 2000). These data provide an important confirmation of the delayed energy release expected when hydrogen is burning via an rp-process. The resemblance of these profiles to theoretical results of Hanawa \& Fujimoto (1984) and Schatz et al. (2001) is striking. 


\subsection{Observational overview of bursts}

In this section we give a brief overview of important observational characteristics of bursts. We will emphasize recent work and specific topics which are relevant in the context of the new discoveries to be described in later sections. We refer to earlier reviews for some of the details we must omit.

Bursts are most commonly observed from the "atoll" subclass of LMXBs with luminosities above $\approx 10^{36} \mathrm{ergs} \mathrm{s}^{-1}$ (Hasinger \& van der Klis 1989; LVT). These systems are accreting at intermediate rates of between $0.01-0.3 \dot{M}_{E d d}$, which is roughly consistent with the accretion rate range of the thermonuclear instabilities described earlier. Interestingly, observations with the WFC on BeppoSAX have recently discovered bursts from 10 LMXBs with persistent X-ray luminosities significantly less than $10^{36}$ ergs s$^{-1}$ (see Cornelisse et al. 2002a; Cornelisse et al. 2002b; Cocchi et al. 2001; §3.3.4). These recently discovered bursts probe the mass accretion rate dependence of thermonuclear burning in a previously unexplored regime. For example, physical processes such as element diffusion, which are not relevant to most bursters, likely become important at such low accretion rates (Wallace, Woosley \& Weaver 1982). We will say some more about these bursts shortly.

\subsubsection{Burst profiles and spectra}

Though the time profiles of X-ray bursts are diverse, they do share several characteristic features. (i) Burst rise times are shorter than their decay times. Rise times are typically $<2 \mathrm{~s}$, but in some cases can be as long as $10 \mathrm{~s}$. Excluding superbursts, which we discuss later, burst decay times range from about $10 \mathrm{~s}$ to several minutes, with most bursts having $10-20 \mathrm{~s}$ decays. (ii) Burst profiles are shorter at higher energies. This is a direct result of cooling of the neutron star surface with time. (iii) Burst profiles are generally smooth, showing an exponential or exponential-like intensity decay.

Swank et al. (1977) and Hoffman, Lewin \& Doty (1977) showed that bursts have thermal (blackbody) spectra. The radius of a blackbody emitting a flux, $F_{b o l}$, at temperature, $T_{b b}$, is; $R_{b b}=d\left(F_{b o l} / \sigma T_{b b}^{4}\right)^{1 / 2}$, where $d$ is the source distance. Measurement of the bolometric fluxes and blackbody temperatures can then be used to infer radii, if the distance is known. Radii inferred in this manner are typically in the range of $\sim 10 \mathrm{~km}$, consistent with cooling of an object having the theoretical size of a neutron star.

Although burst spectra are observationally well described by the Planck function, theoretically they should be harder than a blackbody at the effective temperature of the atmosphere (see London, Howard \& Taam 1984, 1986; Ebisuzaki \& Nakamura 1988; Madej 1991; Titarchuk 1994). This occurs because electron (Compton) scattering is an important opacity source in a neutron star atmosphere. Direct evidence for this effect comes from the peak blackbody temperatures $\left(k T_{b b}>3 \mathrm{keV}\right)$ of some bursts, which are significantly higher than the Eddington effective temperature for reasonable neutron star models (see London, Howard \& Taam 1984; 1986).

\subsubsection{Photospheric radius expansion bursts}

In bright bursts the local X-ray luminosity in the atmosphere may reach the Eddington limit 


$$
L_{E d d}=(4 \pi c G M / \kappa)\left(1-2 G M / c^{2} R\right)^{-1 / 2}=4 \pi R^{2} \sigma T_{e f f}^{4},
$$

where $M, R$, and $\kappa$ are the neutron star mass, radius and atmospheric opacity, respectively, and the photospheric layers can be lifted off the neutron star surface by radiation pressure. Note that $L_{E d d}$ depends on the composition of the accreted atmosphere through the opacity $\kappa$. In these bursts the blackbody temperature decreases while the inferred blackbody radius simultaneously increases. This all happens while the total X-ray flux stays approximately constant. These bursts are called photospheric radius expansion (PRE) bursts. The moment when the photosphere falls back to the neutron star surface (when the temperature is highest) is called "touchdown." Theoretical work indicates that in such bursts the X-ray flux stays within a few percent of the Eddington limit, and the excess energy is efficiently transferred into kinetic energy of the outflow (Hanawa \& Sugimoto 1982; Paczynski \& Anderson 1986; Joss \& Melia 1987; Titarchuk 1994; Shaposhnikov \& Titarchuk 2002). Ebisuzaki \& Nakamura (1988) found evidence for photospheric composition variations when comparing fainter bursts with PRE bursts from the LMXBs 4U 1608-52 and $4 \mathrm{U} 1636-53$. They found differences in the luminosity - color temperature relation between these two classes which they attributed to atmospheric composition changes. They suggested that the luminosity - color temperature relation for faint bursts and PRE bursts could be explained by hydrogen rich and hydrogen poor atmospheres, respectively. They further hypothesized that the hydrogen rich envelope is ejected during bright PRE bursts, and thus the atmospheric composition is pure helium in these cases.

The amount of photospheric uplift can vary dramatically from burst to burst and amongst different sources. In the most powerful bursts the expansion can be large enough to shift the effective temperature of the photosphere entirely below the X-ray band (See Lewin, Vacca \& Basinska 1984; Strohmayer \& Brown 2002). Such events show "precursors" separated from the main part of the burst by the cooling of the photosphere. Other less powerful bursts may show double peaked profiles as only a portion of the flux is shifted out of the X-ray band by the expansion. Figure 3.4 shows several examples of bursts, both with and without PRE, observed with the RXTE/PCA from the LMXB 4U 1728-34.

Since the Eddington luminosity should impose an upper limit to burst fluxes, it was suggested early on that bursts might provide a "standard candle" (van Paradijs 1978), and that they could be used as distance indicators. Van Paradijs (1981) recognized that globular cluster bursters, with independently known distances, would provide an important test of this idea. Subsequent researchers concluded that the brightest (PRE) bursts likely represent a true limiting luminosity, but some uncertainties remained (Lewin 1982; Basinska et al. 1984). Recently Kuulkers et al. (2002a) have reexamined this issue using the extensive sets of bursts observed with RXTE and BeppoSAX/WFC, and a uniform set of globular cluster distances. They conclude that the radius expansion bursts can indeed be regarded as standard candles to within about $15 \%$, and derive a critical luminosity of about $3.8 \times 10^{38} \mathrm{erg} \mathrm{s}^{-1}$, consistent with the Eddington limit for hydrogen-poor matter from a neutron star. Since we do not expect that all LMXBs are pure helium accretors, the fact that PRE bursts from the globular cluster sources have approximately the same peak fluxes 


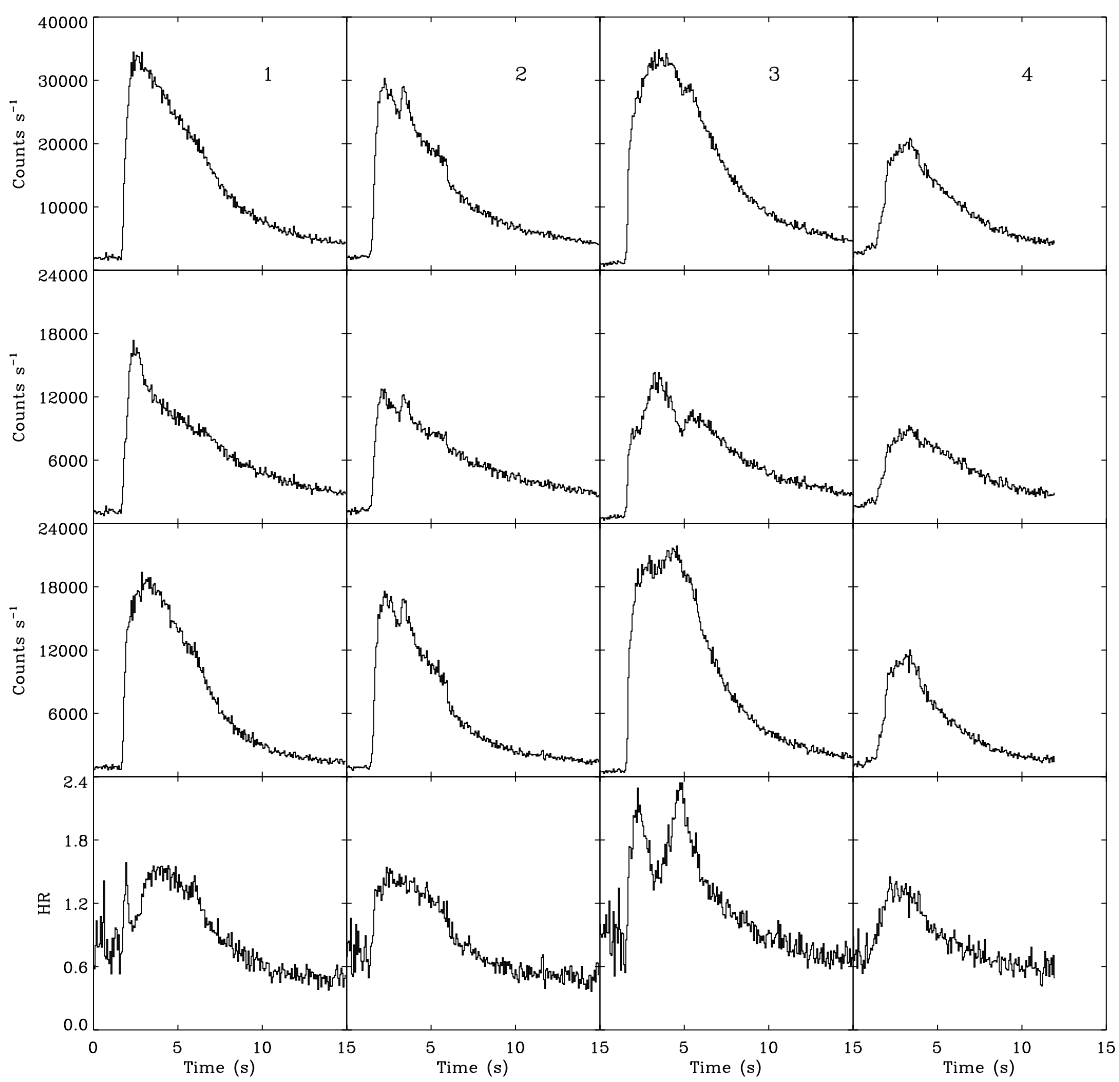

Fig. 3.4. A sample of four X-ray bursts from the LMXB $4 \mathrm{U} 1728-34$ as observed with the RXTE/PCA. Each sequence shows, from top to bottom, the total 2 - $60 \mathrm{keV}$ countrate, the 2 - $6 \mathrm{kev}$ countrate, the $6-30 \mathrm{keV}$ countrate, and the hardness ratio $(6-30 \mathrm{keV}) /(2-6$ $\mathrm{keV})$. Bursts 1 and 3 show clear evidence for PRE based on the hardness ratio evolution.

provides additional support to the idea that in these bursts the hydrogen is blown off in a wind.

Galloway et al. (2002) have recently examined the distribution of peak fluxes of bursts from $4 \mathrm{U} 1728-34$. They find that the peak fluxes of PRE bursts are not constant, but show variations of $\approx 44 \%$. However, they find this variation is correlated with the source spectral state just prior to the bursts, and suggest it may be caused by reprocessing from a precessing, warped disk. Upon removing the correlation they obtain a $3 \%$ variation. They use this result to argue that during radius expansion episodes the emission from the photosphere is largely isotropic. Assuming the peak flux is the Eddington limit appropriate for pure helium they derive a source distance of 5.2 - 5.6 kpc. Smale (1998) and Kuulkers \& van der Klis (2000) used radius expansion bursts observed with RXTE from Cyg X-2 and GX 3+1, respectively, to place new constraints on the distances to these sources.

In principle, observations of PRE bursts provide a means to infer the masses and radii of neutron stars, quantities which have profound implications for our understanding of the equation of state of dense matter (Lattimer \& Prakash 2001). Here 
we will only outline the main ideas, and we refer the reader to LVT for a more detailed discussion. As noted above, the Eddington luminosity at the surface of a neutron star depends only on the stellar mass, $M$, radius, $R$, and the composition of the atmosphere. The above expression (see Equation 3.4) shows that a measurement of the effective temperature, $T_{\text {eff }}$, when the luminosity is Eddington limited, gives a constraint on the mass and radius of the neutron star. Indeed, this constraint is independent of the distance to the source. In practice, however, it is model dependent because the observed color temperature (from, say, a blackbody spectral fit), must be corrected to an effective temperature using an atmosphere model. Typically there are no independent constraints on the atmospheric composition, and so the correct model is uncertain. Measurement of the variation of the Eddington luminosity caused by expansion of the photosphere, and hence a change in the gravitational redshift factor, can also, in principle, be used to determine the gravitational redshift from the neutron star surface (see for example, Damen et al. 1990; van Paradijs et al. 1990; LVT). If the distance to the source is known, then a measure of the burst flux when it is Eddington limited will constrain a slightly different function of $M$ and $R$. Sources with independent distance constraints, such as the globular cluster bursters, can provide, in principle, the best constraints (see Kuulkers 2002a).

Many researchers have used these and related methods to try and constrain neutron star masses and radii (see Fujimoto \& Taam 1986; Ebisuzaki 1987; Sztajno et al. 1987; van Paradijs \& Lewin 1987; Chevalier \& Ilovaisky 1990; Kaminker et al. 1990; Damen et al. 1990; van Paradijs et al. 1990; Haberl \& Titarchuk 1995). In general, the constraints are consistent with a range of neutron star mass - radius relations, but because of systematic uncertainties in the spectra, composition, and burst flux isotropy, they are not generally precise enough to unambiguously constrain the neutron star equation of state. Such efforts have continued using the higher signal to noise data from RXTE (see for example Smale 2001; Strohmayer et al. 1998; Titarchuk \& Shaposhnikov 2002), however, systematic uncertainties remain.

\subsubsection{Recent progress in burst spectroscopy}

The most reliable way to overcome the systematic uncertainties in the interpretation of continuum spectra from bursts is to detect line features from the neutron star surface, and thereby obtain a direct measurement of the gravitational redshift, $1+z=\left(1-2 G M / c^{2} R\right)^{-1 / 2}$. Waki et al. (1984), and Nakamura, Inoue \& Tanaka (1988) reported absorption lines at $4.1 \mathrm{keV}$ in TENMA data from the LMXBs 4U 1636-53, and 4U 1608-52. Magnier et al (1989) found a similar feature from $4 \mathrm{U}$ 1747-214 using EXOSAT data. Waki et al. (1984) identified the line with the helium-like iron Ly $\alpha$ transition at $6.7 \mathrm{keV}$, but gravitationally redshifted from the neutron star surface. The high rmimplied equivalent widths (hundreds of $\mathrm{eV}$ ), however, led others to suggest an origin outside the neutron star atmosphere, perhaps in the accretion flow (see Day, Fabian \& Ross 1992; Foster, Fabian \& Ross 1987; Pinto, Taam \& Laming 1991). More recent observations, for example, with ASCA, RXTE and BeppoSAX, have generally not confirmed the presence of such lines in burst spectra. The interpretation, and reality, of these features has therefore remained controversial.

In spite of this controversy, reports of deviations from blackbody spectra during 
some bursts continue to appear. Kuulkers et al. (2002b) found systematic deviations from blackbody spectra in RXTE data of bursts from the high accretion rate Z source GX $17+2$. The residuals were most significant during the radius expansion and contraction phases. These variations are quite similar to those described by van Paradijs et al. (1990) for a PRE burst from 4U 2129+11, and, more recently, by Franco \& Strohmayer (1999) for a burst from 4U 1820-30 (see also Kuulkers et al. 2002a for a discussion). Highly significant discrete spectral components, which could be modelled as an $\approx 6.4 \mathrm{keV}$ emission line and $\approx 8 \mathrm{keV}$ absorption edge have recently been found in RXTE/PCA spectra during a superburst from $4 \mathrm{U}$ 1820-30 (see Strohmayer \& Brown 2002). It seems likely that these features may result from reprocessing (disk reflection) and fluorescence in the accretion disk, or perhaps are formed in the burst-driven wind. It is not yet known if the features observed during PRE bursts are directly related to the features observed during the $4 \mathrm{U} 1820-30$ superburst. Until their identifications are more secure it will be difficult to infer neutron star properties with them, however, these recent results have established convincingly that some X-ray burst spectra do have discrete lines. Observations with sufficient collecting area at higher spectral resolution will likely provide the breakthrough needed for reliable interpretation of these features.

The high spectral resolution capabilities of Chandra and XMM/Newton are providing new tools to study neutron star spectra, however, with one recent exception (see Sanwal et al. 2002; Mereghetti et al. 2003), the high resolution spectra of isolated neutron stars have been frustratingly devoid of line features (see for example, Walter \& Lattimer 2002; Burwitz et al. 2001; Drake et al. 2002; Pavlov et al. 2001). Indeed, bursters may prove to be more promising targets for line searches because of the mix of heavy elements constantly being provided by accretion. For example, Cottam, Paerels \& Mendez (2002) have recently reported evidence for redshifted absorption lines in XMM/Newton Reflection Grating Spectrometer (RGS) data of bursts from EXO 0748-676. They co-added data from 28 bursts in order to increase the sensitivity to narrow lines. After modelling the continuum and line features thought to be produced in the highly ionized gas surrounding the neutron star (see Cottam et al. 2001), they found several features which were unaccounted for by their best model. Figure 3.5, from Cottam, Paerels \& Mendez (2002), shows their RGS spectra and best model. They interpreted the unmodelled features near 13 and 14 Angstroms as redshifted absorption lines of the $\mathrm{n}=2-3$ transitions of hydrogen-like Fe (Fe XXVI) in the early (hotter) part of the bursts, and the same transitions in the helium-like ion in the later (cooler) portions of the bursts. The implied neutron star surface redshift in each case was $z=0.35$, and is consistent with modern neutron star equations of state with reasonable masses (Lattimer \& Prakash 2001). It also implies that the neutron star surface is inside the last stable circular orbit (Kluzniak \& Wagoner 1985).

\subsubsection{Recurrence intervals and the $\dot{M}$ dependences}

We previously discussed the one case where the observed type I bursts matched theory well. However, most bursters are not so easy to understand, and we discuss that more typical case here as it starts to point to the possibility of nonspherically symmetric behavior. In a particular burning regime, we expect that the 


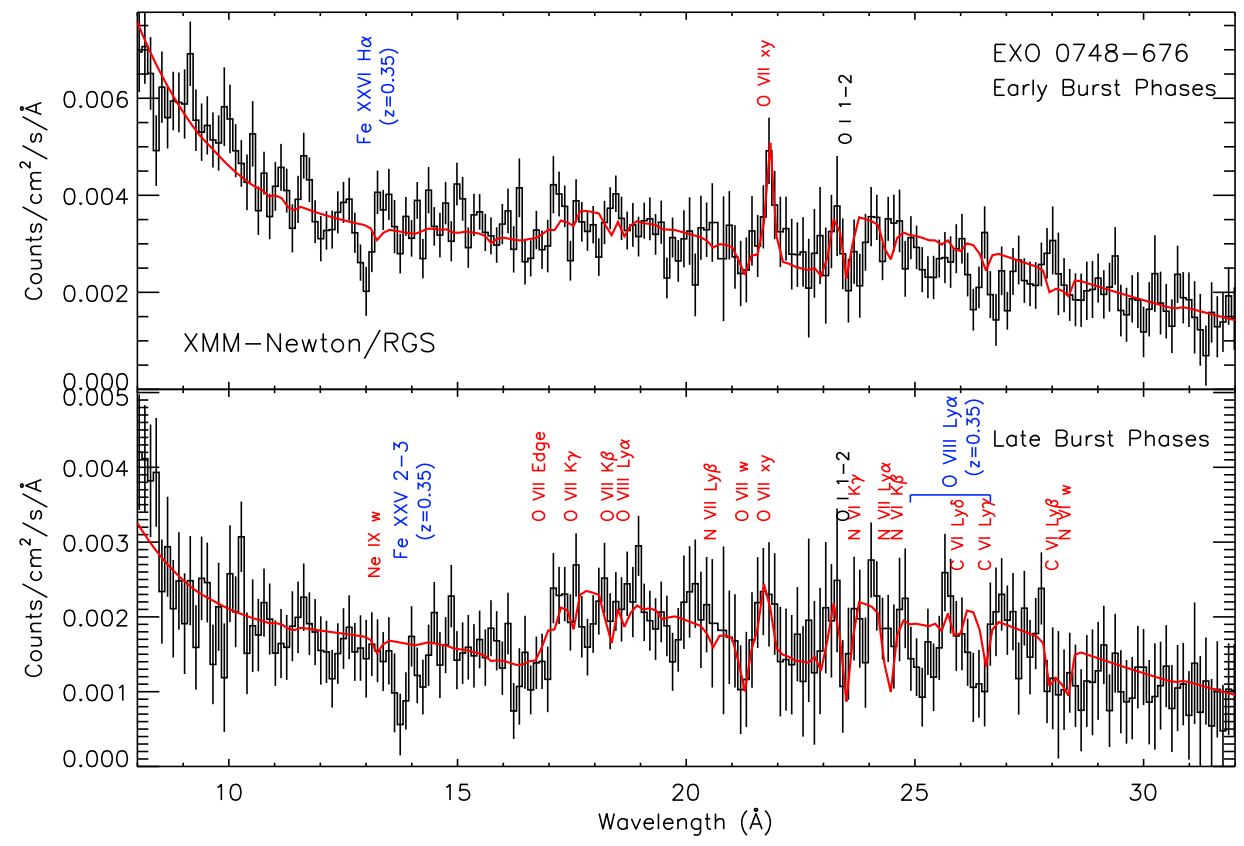

Fig. 3.5. High resolution X-ray spectra of bursts from the LMXB EXO 0748-676. The XMM-Newton Reflection Grating Spectrometer (RGS) data from the early (top) and late (bottom) phases of the sum of $28 \mathrm{X}$-ray bursts are shown. The solid curve shows the best model of continuum and line features due to ionized gas in the vicinity of the neutron star. The unmodelled dips near 13 (top) and 14 (bottom) Angstroms are consistent with redshifted lines of Fe XXVI from the neutron star surface (after Cottam, Paerels \& Mendez 2002).

time between bursts should decrease as $\dot{M}$ increases since it takes less time to accumulate the critical amount of fuel at a higher $\dot{M}$. Exactly the opposite behavior was observed from many low accretion rate $\left(\dot{M}<10^{-9} M_{\odot} \mathrm{yr}^{-1}\right)$ NSs. A particularly good example is $4 \mathrm{U} 1705-44$, where the recurrence time increased by a factor of $\approx 4$ when $\dot{M}$ increased by a factor of $\approx 2$ (Langmeier et al. 1987, Gottwald et al. 1989). If the star is accreting matter with $Z_{C N O}=0.01$ then these accretion rates are at the boundary between unstable helium ignition in a hydrogen-rich environment at high $\dot{M}$ and unstable pure helium ignition at lower $\dot{M}$. The expected change in burst behavior as $\dot{M}$ increases would then be to more energetic and more frequent bursts. This was not observed.

Other NSs showed similar behavior. Van Paradijs, Penninx \& Lewin (1988) tabulated this effect for many bursters and concluded: "This suggests that continuous stable burning of a sizeable fraction of the nuclear fuel is a general phenomenon on the surface of accreting neutron stars. This fraction is apparently a gradually increasing function of $\dot{M}$ ". The following trends were found as $\dot{M}$ increases: 
- The recurrence time increases from $2-4$ hours to 10 hours.

- The bursts burn less of the accumulated fuel, with $\alpha$ increasing from $\approx 40$ to $>100$.

- The duration of the bursts decreases from $\approx 30 \mathrm{~s}$ to $\sim 5 \mathrm{~s}$.

More recent $R X T E$ observations found the same trend in KS 1731-260 (Muno et al. 2000), but not in 4U 1728-34 (Franco 2001; van Straaten et al. 2001).

The low $\dot{M}$ bursts appear to be mixed H/He burning with a large $\mathrm{H}$ mass fraction (i.e., energetic and of long duration from the rp-process) whereas the high $\dot{M}$ bursts look more $\mathrm{He}$ dominated with less $\mathrm{H}$ present (i.e., not so energetic, recurrence times long enough to burn $\mathrm{H}$ while accumulating, and short duration due to the lack of much energy release from the rp-process). The simplest explanation is to say that the NS is near the transition from the low $\dot{M}$ mixed burning regime (1 in §3.2.1) to the higher $\dot{M}$ pure helium burning (noted as 2 in $\S 3.2 .1$ ). This would require that the NSs are accreting at $\approx 10^{-10} M_{\odot} \mathrm{yr}^{-1}$ in the lower $\dot{M}$ state and about a factor of $4-5$ higher in the high $\dot{M}$ state. However, these estimates are very far away from that observed.

Van Paradijs et al. (1988) used the ratio of the persistent flux to the flux during Eddington limited PRE bursts as a measure of $\dot{M}$ in units of the Eddington accretion rate. They showed that most bursters accrete at rates $\dot{M} \approx(3-30) \times 10^{-10} M_{\odot} \mathrm{yr}^{-1}$, at least a factor of three (and typically more) higher than the calculated rate where such a transition should occur. Moreover, if the accretion rates were as low as needed, the recurrence times for the mixed hydrogen/helium burning would be about 30 hours, rather than the observed $2-4$ hours. Fujimoto et al. (1987) discussed in some detail the challenges these observations present to a spherically symmetric model, while Bildsten (1995) attempted to resolve this by having much of the thermally unstable burning occur via slow deflagration fronts that lead to very low frequency noise and not Type I bursts (see also Yu et al. 1999). Whether this is possible on rapidly rotating NSs is uncertain (Spitkovsky, Levin \& Ushomirsky 2002).

Much of this puzzle might be resolved by allowing the fresh material to only cover a fraction of the star prior to igniting (Bildsten 2000). There are strong observational clues that this may be happening (e.g. Marshall 1982), as the other clear trend found by EXOSAT was an increase in the apparent black-body radius $\left(R_{b b}\right)$ as $\dot{M}$ increased. This parameter is found by spectral fitting in the decaying tail of the Type I bursts and, though susceptible to absolute spectral corrections, can likely be trusted for relative sizes (LVT). In a similar vein, van der Klis et al. (1990) found that the temperature of the burst at the moment when the flux was one-tenth the Eddington limit decreased as $\dot{M}$ increased (hence a larger area) for the Atoll source $4 \mathrm{U}$ 1636-53. In total, these observations suggest the possibility that the covered area increases fast enough with increasing $\dot{M}$ that the accretion rate per unit area actually decreases.

By interpreting the measured $R_{b b}$ as an indication of the fraction of the star that is covered by freshly accreted fuel, the quantity $\dot{m}=\dot{M} / 4 \pi R_{b b}^{2}$ can be calculated using $F_{x}=G M \dot{M} / 4 \pi d^{2} R$ and gives $\dot{m} \approx\left(F_{x} R / G M\right)\left(d / R_{b b}\right)^{2}$. Bildsten (2000) has argued that the radius increase can offset the $\dot{M}$ increase in the context of data from the burster EXO 0748-676 (Gottwald et al. 1986; see Figure 1 in Bildsten 2000). 
Where does the material arrive on the NS? We know that these NSs accrete from a disk formed in the Roche lobe overflow of the stellar companion, however, there are still debates about the "final plunge" onto the NS surface. Some advocate that a magnetic field controls the final infall, while others prefer an accretion disk boundary layer. These arguments must also take into account the possible presence of an accretion gap between the inner disk edge and the stellar surface (Kluzniak, Michelson \& Wagoner 1990, Kluzniak \& Wilson 1991), as the gravitational redshift measurement reported by Cottam et al. (2002) implies that the NS surface is inside the last stable orbit. If material is placed in the equatorial belt, it is not clear that it will stay there very long. If angular momentum was not an issue, the lighter accreted fuel (relative to the ashes) would cover the whole star quickly. However, on these rapidly rotating NSs, the fresh matter added at the equator must lose angular momentum to get to the pole. This competition (namely understanding the spreading of a lighter fluid on a rotating star) has been recently investigated by Inogamov \& Sunyaev (1999).

Another remaining conundrum is the burst behavior of the bright $\mathrm{Z}$ sources ( $\mathrm{Sco}$ X-1, Cyg X-2, GX 5-1, GX 17+2, GX 340+0, GX 349+2) which are accreting at $3 \times 10^{-9}-2 \times 10^{-8} M_{\odot} \mathrm{yr}^{-1}$. At the very upper end of this range, the burning could be thermally stable, however, if unstable, they would clearly be in the regime where the bursts are regularly spaced and of long duration due to mixed hydrogen/helium burning (noted as 3 above and as exhibited by GS 1826-24). However, this is not typically seen, rather, bursts are rare and are clearly not responsible for burning all of the accreted fuel. Though some of the recently studied bursts from GX $17+2$ (Kuulkers et al. 2002b) do look as expected, not all are easy to understand and we refer the interested reader to Kuulkers et al. (2002b) for an excellent summary discussion of the current observational situation at high $\dot{M}$ 's.

Most of the Type I bursts we have discussed occur when the neutron star is accreting at a rate in excess of $10^{-10} M_{\odot} \mathrm{yr}^{-1}$. Recently, however, the BeppoSAX WFCs have discovered a number of new thermonuclear burst sources from which no persistent X-ray flux could be detected down to the $10^{-10} \mathrm{erg} \mathrm{cm}^{-2} \mathrm{~s}^{-1}$ flux limit of the WFCs (see for example, Cornelisse et al. 2002a; Cocchi et al. 2002; in 't Zand et al. 2002). If these objects are closer than $10 \mathrm{kpc}$ then their accretion rate when bursting was less than $10^{-10} M_{\odot} \mathrm{yr}^{-1}$ (or $L<10^{36} \mathrm{erg} \mathrm{s}^{-1}$ ).

At other epochs, some of these sources were observed by ROSAT at luminosities $\approx 10^{35} \mathrm{erg} \mathrm{s}^{-1}$, whereas later Chandra observations of several of them revealed persistent luminosities (or upper limits) in the $10^{32-33} \mathrm{erg} \mathrm{s}^{-1}$ range (Cornelisse et al. 2002b), consistent with flux levels seen from neutron star X-ray transients in quiescence (see for example Rutledge et al. 2001). Cocchi et al. (2001) and Cornelisse et al. (2002b) have suggested that these bursters may be either a new class of low persistent emission bursters (in which case there is a huge underlying population) or weak transients with time-averaged accretion rates $<10^{-11} M_{\odot} \mathrm{yr}^{-1}$ (like SAX J1808.4-3658) that just happen to be further away.

Regardless of whether these systems are constantly accreting at such a low rate or are weak transients in outburst, what is important for this discussion is that the bursts occur when the accretion rate is clearly less than $10^{-10} M_{\odot} \mathrm{yr}^{-1}$. Presuming complete covering of the neutron star, the hydrogen burning would be unstable at these accretion rates (regime 1 in $\S 3.2 .1$ ) and the unstable ignition should lead to long 
$(100-1000 \mathrm{~s})$, infrequent bursts (recurrence times of $\approx$ weeks). Some of the recently discovered sources appear to be in this regime (Kaptein et al. 2000; in 't Zand et al. 2002), however, it is not known if all these "burst-only" sources can be explained this way. In fact, it seems unlikely, since some of the observed bursts are short and therefore inconsistent with the standard low accretion rate theory. It is conceivable that our understanding of these sources may require additional, important physics, such as element diffusion, which is not important for higher accretion rates but becomes more relevant at these rates (Wallace et al. 1982, Bildsten, Salpeter \& Wasserman 1993).

\subsection{Millisecond variability during X-ray bursts}

Early theoretical studies noted the likely importance of spreading of the thermonuclear burning front around the neutron star surface (see for example, Joss 1978). Because nuclear fuel is burned in a time much shorter than it takes to accrete a critical pile it is unlikely that ignition conditions will be achieved over the entire surface simultaneously (Shara 1982). It appears more likely that burning is initiated locally and then spreads laterally, eventually engulfing all fuel-loaded parts of the neutron star. For conditions most prevalent in burst sources, the front may spread via convective deflagration, at lateral speeds of up to $\approx 5 \times 10^{6} \mathrm{~cm} \mathrm{~s}^{-1}$ (see Fryxell \& Woosley 1982; Hanawa \& Fujimoto 1984; Nozakura, Ikeuchi \& Fujimoto 1984; Bildsten 1995). Such speeds can account for the sub-second rise times of some bursts, but the time required for burning to engulf the entire star is still long compared to the spin periods of accreting LMXB neutron stars (milliseconds). Moreover, if the burning front is not strongly convective, then a patchy distribution of nuclear fuel is possible (see Bildsten 1995). These considerations suggest that during bursts the rotation of the neutron star can modulate the inhomogeneous or localized burning regions, perhaps allowing for direct observation of the spin of the neutron star.

Mason et al. (1980) reported a $36.4 \mathrm{~Hz}$ pulsation in an optical burst from $4 \mathrm{U}$ 1254-690, and Sadeh (1982) claimed detection of a 12 ms modulation in a burst from $4 \mathrm{U}$ 1728-34 with HEAO-1, but these periods were never confirmed by subsequent observations. Murakami et al. (1987) reported $1.5 \mathrm{~Hz}$ oscillations during the PRE phase of a burst from $4 \mathrm{U} 1608-52$. These oscillations might conceivably be caused by oscillations of the photospheric radius at constant luminosity (Lapidus et al. 1994). Schoelkopf \& Kelley (1991) reported detection at the $\approx 4 \sigma$ level of a $7.6 \mathrm{~Hz}$ oscillation in Einstein Monitor Proportional Counter data during a burst from Aql X-1. They suggested that rotation of the neutron star with a non-uniform surface brightness might be responsible, but the signal has not been seen in other bursts. Jongert \& van der Klis (1996) searched for high frequency variability in bursts observed with the EXOSAT observatory. They averaged power spectra from multiple bursts from the same source to increase sensitivity, but also found no significant periods. They

placed upper limits on average modulation amplitudes in the $\sim 100 \mathrm{~Hz}$ range of between $5-10 \%$.

\subsubsection{Overview of burst oscillations}

An exciting development in the past decade has been the discovery of high frequency $(300-600 \mathrm{~Hz})$ X-ray brightness oscillations during bursts. These mod- 


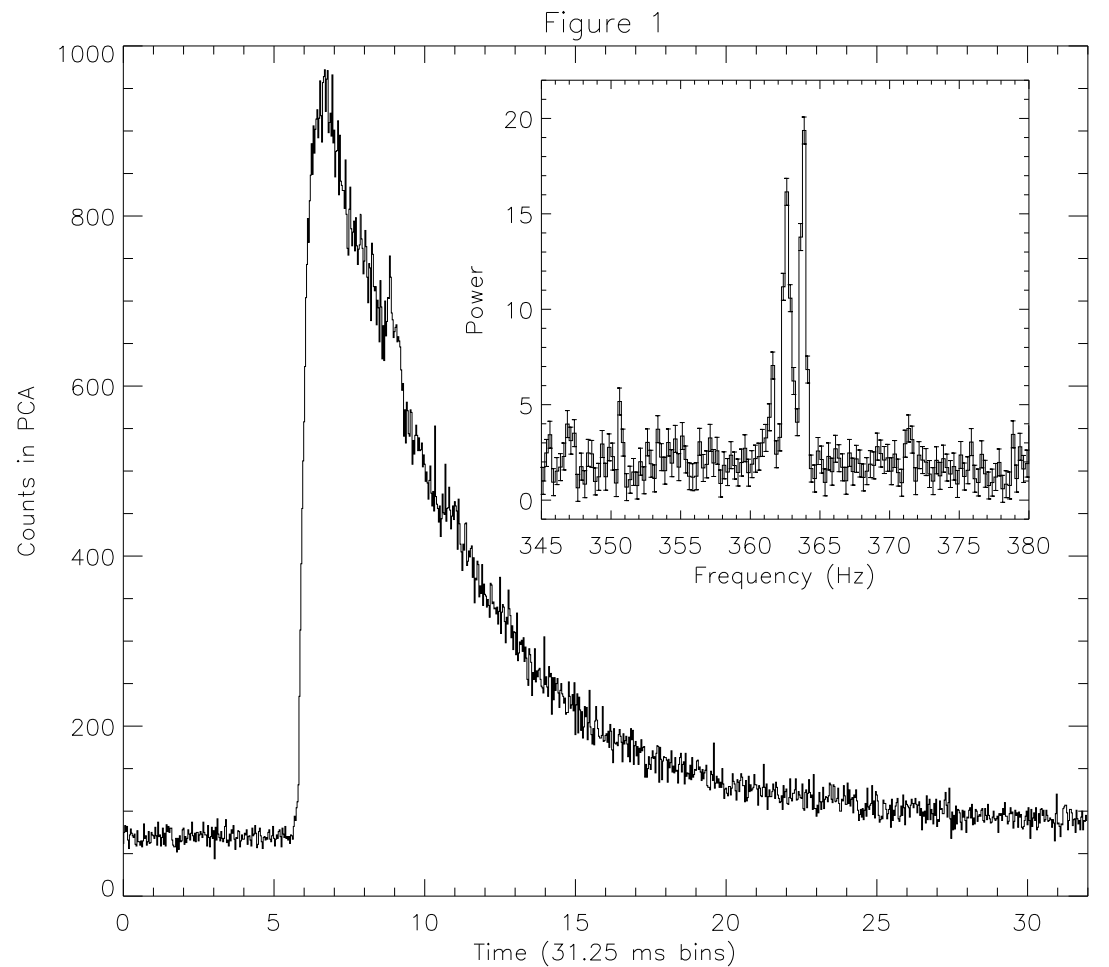

Fig. 3.6. An X-ray burst from $4 \mathrm{U} 1728-34$ observed with the PCA onboard RXTE. The main panel shows the X-ray counts observed by the PCA in $(1 / 32) \mathrm{s}$ bins. The inset panel shows the power spectrum in the vicinity of $363 \mathrm{~Hz}$ (after Strohmayer et al. 1996).

ulations are now commonly called "burst oscillations." They were first discovered with the PCA onboard RXTE in bursts from the LMXB 4U 1728-34 (Strohmayer et al. 1996). As of this writing, burst oscillation detections have been claimed for an additional ten sources, one of which is the $401 \mathrm{~Hz}$ accreting millisecond pulsar SAX J1808.4-3658 (in 't Zand et al. 2001; Wijnands et al. 2002), whose spin period is precisely known. Table 3.1 provides a catalog of the known burst oscillation sources, summarizes some of their salient properties, and provides references to the relevant literature.

Burst oscillations were first discovered in Fourier power spectra computed from high time resolution lightcurves of entire bursts. Figure 3.6 shows an example of a Fourier power density spectrum of a burst from 4U 1728-34 with a burst oscillation at $363 \mathrm{~Hz}$ (after Strohmayer et al. 1996). Although many detailed questions remain, there is now little doubt that spin modulation of the X-ray burst flux is the basic mechanism responsible for these oscillations. The discovery of burst oscillations was closely linked with the discovery of millisecond variability ( $\mathrm{kHz}$ QPOs) in the persistent X-ray flux from accreting neutron stars. Such oscillations, and some of their inferred connections to burst oscillations, are reviewed in Chapter 2 by van 
Table 3.1. Burst oscillation sources and properties

\begin{tabular}{ccccc}
\hline \hline Source & Frequency $(\mathrm{Hz})$ & $\Delta \nu_{q p o}^{2}$ & $P_{\text {orb }}(\mathrm{hr})^{3}$ & References $^{1}$ \\
\hline $4 \mathrm{U} 1728-34$ & 363 & $280-363$ & $?$ & $1,2,3,4,5,13,14$ \\
$4 \mathrm{U} 1636-53$ & 581 & $250-320$ & 3.8 & $6,7,25,26,29$ \\
KS 1731-260 & 524 & 260 & $?$ & $10,11,12$ \\
Galactic Center & 589 & $?$ & $?$ & 15 \\
Aql X-1 & 549 & $?$ & 19.0 & 16,17 \\
4U 1702-429 & 330 & $315-344$ & $?$ & 4,9 \\
MXB 1658-298 & 567 & $?$ & 7.1 & 18,27 \\
4U 1916-053 & 270 & $290-348$ & 0.83 & 19,20 \\
4U 1608-52 & 619 & $225-325$ & $?$ & 8,21 \\
SAX J1808.4-3658 & 401 & $?$ & 2.0 & $22,23,28$ \\
SAX J1750.8-2980 & 601 & $?$ & $?$ & 24 \\
\hline \hline
\end{tabular}

1 (1) Strohmayer et al. (1996); (2) Strohmayer, Zhang, \& Swank (1997); (3) Mendez \& van der Klis (1999); (4) Strohmayer \& Markwardt (1999); (5) Strohmayer et al. (1998b); (6) Strohmayer et al. (1998a); (7) Miller (1999); (8) Mendez et al. (1998); (9) Markwardt, Strohmayer \& Swank (1999) (10) Smith, Morgan, \& Bradt (1997); (11) Wijnands \& van der Klis (1997); (12) Muno et al. (2000); (13) van Straaten et al. (2000); (14) Franco (2000); (15) Strohmayer et al (1997); (16) Zhang et al. (1998); (17) Ford (1999); (18) Wijnands, Strohmayer \& Franco (2001); (19) Boirin et al. (2000); (20) Galloway et al. (2001); (21) Chakrabarty (2000); (22) in 't Zand et al. (2001); (23) Ford (2000); (24) Kaaret et al (2002); (25) Giles et al. (2002); (26) Strohmayer \& Markwardt (2002); (27) Wijnands et al. (2002); (28) Wijnands et al. (2002); (29) Jonker, Mendez \& van der Klis (2002).

${ }^{2}$ The frequency separation between pairs of $\mathrm{kHz}$ QPO in $\mathrm{Hz}$, if known.

${ }^{3}$ Orbital period of the system, in hours, if known.

der Klis. Therefore, we will only review the observational properties and current theoretical understanding of burst oscillations and will not discuss in any great detail the possible connections between the two phenomena.

\subsubsection{Oscillations during the burst rise}

In some bursts oscillations are detected during the rising portion of the burst time profile. Indeed, detections can be made within a few tenths of a second after a significant rise in the X-ray flux is seen (see Strohmayer et al. 1988a; Miller 1999). Such oscillations can have very large amplitudes. Strohmayer, Zhang \& Swank (1997) found that some bursts from 4U 1728-34 show oscillation amplitudes as large as $43 \%$ within $0.1 \mathrm{~s}$ of the onset of the burst. Strohmayer et al. (1998a) found bursts from $4 \mathrm{U} 1636-53$ with modulations at onset of $\approx 75 \%$ (see Figure 3.7). These early studies also found that the modulation amplitude is anti-correlated with the X-ray intensity during the rise. That is, the amplitude drops as the flux increases toward maximum. This behavior is consistent with simple expectations for spin modulation of an initially localized X-ray "hot spot" which expands in $\sim 1$ $\mathrm{s}$ to engulf the neutron star. That is, the amplitude is largest when the hot spot is smallest, at onset, and then decreases as the spot engulfs the star and the flux 


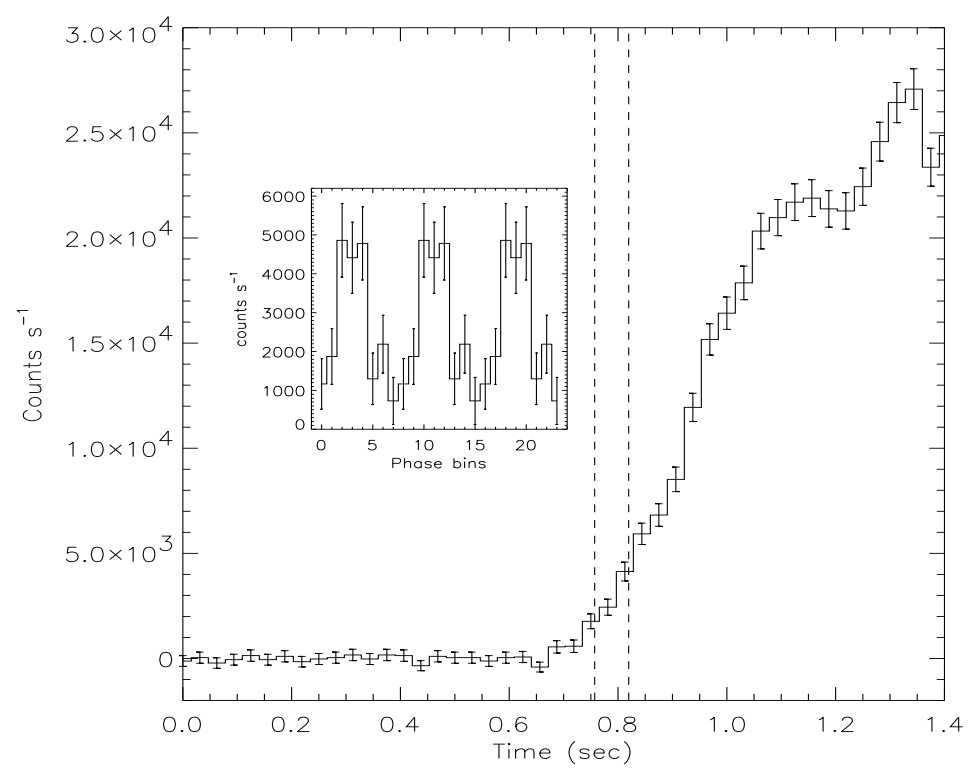

Fig. 3.7. X-ray timing evidence indicating a spreading hot spot at the onset of thermonuclear bursts. The main panel shows a burst from $4 \mathrm{U} 1636-53$ with large amplitude, $581 \mathrm{~Hz}$ oscillations on the rising edge of the profile. The inset shows the pulse profile during the interval marked by the vertical dashed lines. The pulse profile is repeated $3 \times$ for clarity. Note the large amplitude of the oscillation. (after Strohmayer et al. 1998a).

increases (see Nath, Strohmayer \& Swank 2002). Time resolved X-ray spectroscopy during bursts provides additional evidence for localized X-ray emission near burst onset. For example, Strohmayer, Zhang \& Swank (1997) tracked the evolution of the bolometric flux and blackbody temperature during bursts from $4 \mathrm{U} 1728-34$. For a spherical blackbody source, the quantity $F_{b o l}^{1 / 4} / k T_{b b}$ is proportional to the square root of the emitting area (see also the discussions in $\S 3.3 .1$ and 3.3.4). They found that this inferred surface area was smallest at onset and then increased as the burst flux increased during the rise. The inferred area then stayed approximately constant as the flux declined (see Figure 3.8).

\subsubsection{Oscillations in the decay phase}

Oscillations are also observed at late times in the cooling or decay phase of bursts. In many cases oscillations are seen after "touchdown" in PRE bursts (see for example, Smith, Morgan \& Bradt 1996; Muno et al. 2000), however, bursts without PRE can also show oscillations which persist during the cooling phase (Franco 2001; van Straaten 2001). Typically the amplitude of oscillations seen in the cooling phase is lower than observed during burst rise, but amplitudes as large as $15 \%$ (rms) have been observed. Some bursts show oscillations both on the rise and in the cooling phase (see van Straaten 2001; Franco 2001), however, oscillations are not detected during PRE phases, only before and after the PRE episode (see Muno et al. 2002). This provides additional evidence that the modulations are associated with processes on the neutron star surface. In bursts where pulse trains are detected during the rise 


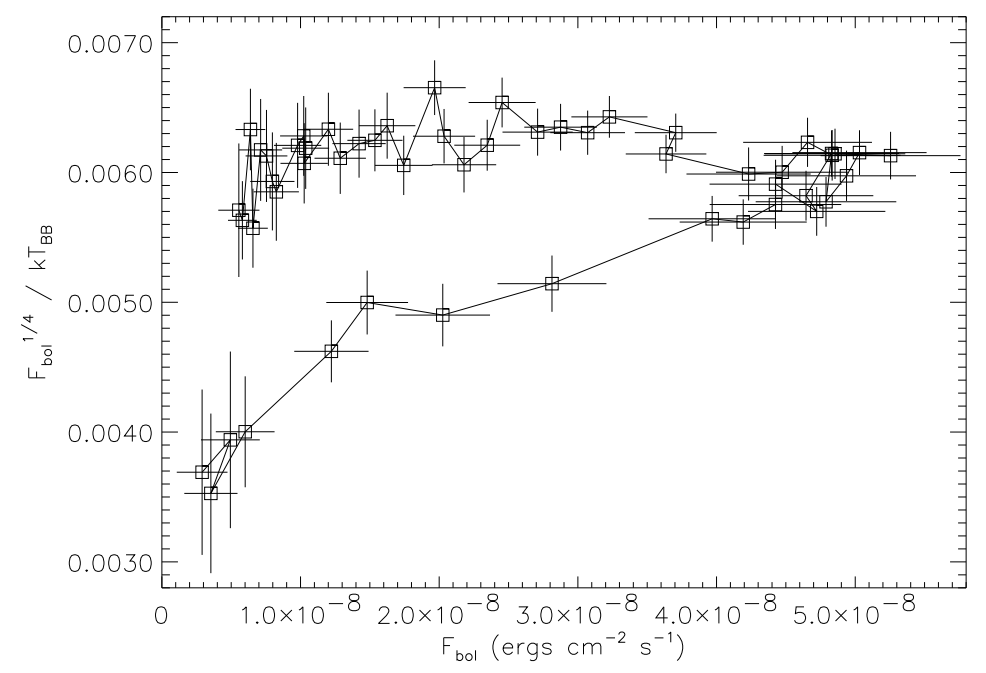

Fig. 3.8. X-ray spectral evidence indicating a spreading hot spot at the onset of thermonuclear bursts. The plot shows the time evolution of the inferred surface area in a burst from $4 \mathrm{U}$ 1728-34. The evolution is from lower left to upper right, and then horizontally to the left (after Strohmayer, Zhang \& Swank 1997).

and decay they can sometimes be observed for as long as $\approx 12$ seconds. The presence of strong oscillations after the nuclear burning has presumably engulfed the entire neutron star is difficult to account for in the simplest expanding hot spot scenarios. This has motivated a number of recent theoretical ideas to explain the persistence of burst oscillations. We will discuss some of these ideas shortly.

\subsubsection{Frequency evolution of burst oscillations}

The oscillation frequency during a burst is typically not constant. Most commonly the frequency increases as the burst progesses, that is, the evolution can be characterized as a "chirp." Most bursts show spin-up toward some limiting, or asymptotic frequency, however, there are exceptions to this rule of thumb. For example, Strohmayer (1999) and Miller (2000) identified a burst from 4U 1636-53 with spin down in the cooling tail. This burst also showed an unusually long thermal tail which may have been related to a "reheating" episode having some connection to the spin down. Muno et al. (2000) found spin down in a burst from KS 1731-260, however, they found no evidence for unusual flux enhancements or spectral variations during the episode. Spin downs are apparently rare. In a recent study, Muno et al. (2002) found some evidence for them in only 3 of 68 bursts examined. The observed frequency drifts are generally $<1 \%$ of the mean frequency, and bursts which have detectable pulsations during the rising phase show the largest frequency shifts. This indicates that the process responsible for the frequency evolution begins with the start of a burst, and not when oscillations are first detected within a burst (Muno et al. 2002).

Strohmayer \& Markwardt (1999) studied the frequency evolution in bursts from $4 \mathrm{U} 1702-429$ and $4 \mathrm{U} 1728-34$. They found the frequency in these bursts could be 
modelled as a smooth exponential recovery of the form, $\nu(t)=\nu_{0}\left(1-\delta_{\nu} e^{-t / \tau}\right)$, where $\nu_{0}, \delta_{\nu}$, and $\tau$ are the asymptotic frequency, the fractional frequency drift, and the recovery timescale, respectively. With this form they were able to recover coherent signals, with coherence values, $Q \equiv \nu_{0} / \Delta \nu_{0}>4,500$ in some bursts. Figure 3.9 shows an example of a burst from $4 \mathrm{U} 1702-429$ with exponential frequency evolution. These results support the existence of a reference frame on the neutron star, perhaps the nuclear burning layer, in which the oscillations are coherent or nearly so. This frame, however, cannot be rigidly connected to the bulk of the neutron star, because the torque required to change the spin frequency of the star by $\approx 1 \%$ in only 10 seconds is unphysically large. This implies the existence of shearing in the surface layers of the neutron star. In the exponential model, the total amount of phase shearing is simply $\phi_{\text {shear }}=\nu_{0} \delta_{\nu}\left(1-e^{-T / \tau}\right)$, where $T$ is the length of the pulse train. For typical bursts this value ranges from about $4-8$, suggesting that the burning layer "slips" this many revolutions over the underlying neutron star during the duration of the pulsations. The amount of phase shearing has implications for the surface magnetic field strength, as a sufficiently strong field will enforce co-rotation (Cumming, Zweibel \& Bildsten 2001).

Muno et al. (2000) carried out a detailed study of the frequency evolution in burst oscillations from KS 1731-260. They used pulsar timing techniques to compute phase-connected timing solutions in order to study the functional form of the frequency evolution. They found that the phase evolution can usually be modelled as a polynomial, and that the exponential relaxation model was adequate for many bursts. More recently, Muno and colleagues (2002) have explored the frequency evolution in a larger sample of bursts (68) from several sources. They confirm many of their earlier findings, however, they do find a subset of bursts for which simple 2nd and 3rd order polynomials are insufficient to explain the phase evolution. In these bursts there is evidence for phase jitter on timescales of seconds. Figure 3.10 shows several examples of burst oscillations with complex phase evolution. They suggest this may indicate the presence of some instability in the mechanism which generates the oscillation. One idea is that two signals, or modes, with nearly equal frequency may be simultaneously present (see also Miller 1999). Another possibility is that phase jumps occur on relatively short timescales (see Strohmayer 2001). These behaviors, though infrequent, may provide important clues to the physical mechanism which produces the observed modulations, particularly in the cooling phase.

Strohmayer et. al (1997) argued that the observed frequency evolution results from angular momentum conservation of the thermonuclear shell. Burst-induced heating expands the shell, increasing its rotational moment of inertia and slowing its spin rate. Near burst onset the shell is thickest and thus the observed frequency lowest. The shell spins back up as it cools and recouples to the underlying neutron star. Calculations indicate that the $\sim 10 \mathrm{~m}$ thick pre-burst shell can expand to $\sim 30 \mathrm{~m}$ during the flash (see Joss 1978; Bildsten 1995; Cumming \& Bildsten 2000), which gives a frequency shift of $\approx 2 \nu_{\text {spin }}(20 \mathrm{~m} / R)$, where $\nu_{\text {spin }}$ and $R$ are the stellar spin frequency and radius, respectively. For typical burst oscillation frequencies this gives a shift of $\sim 2 \mathrm{~Hz}$, similar to that observed. However, Galloway et al. (2000) reported a $3.5 \mathrm{~Hz}$ frequency shift in a burst from $4 \mathrm{U} 1916-053$ with $272 \mathrm{~Hz}$ oscillations. They suggested that such a large change, $\sim 1.3 \%$ might be inconsistent with expansion 


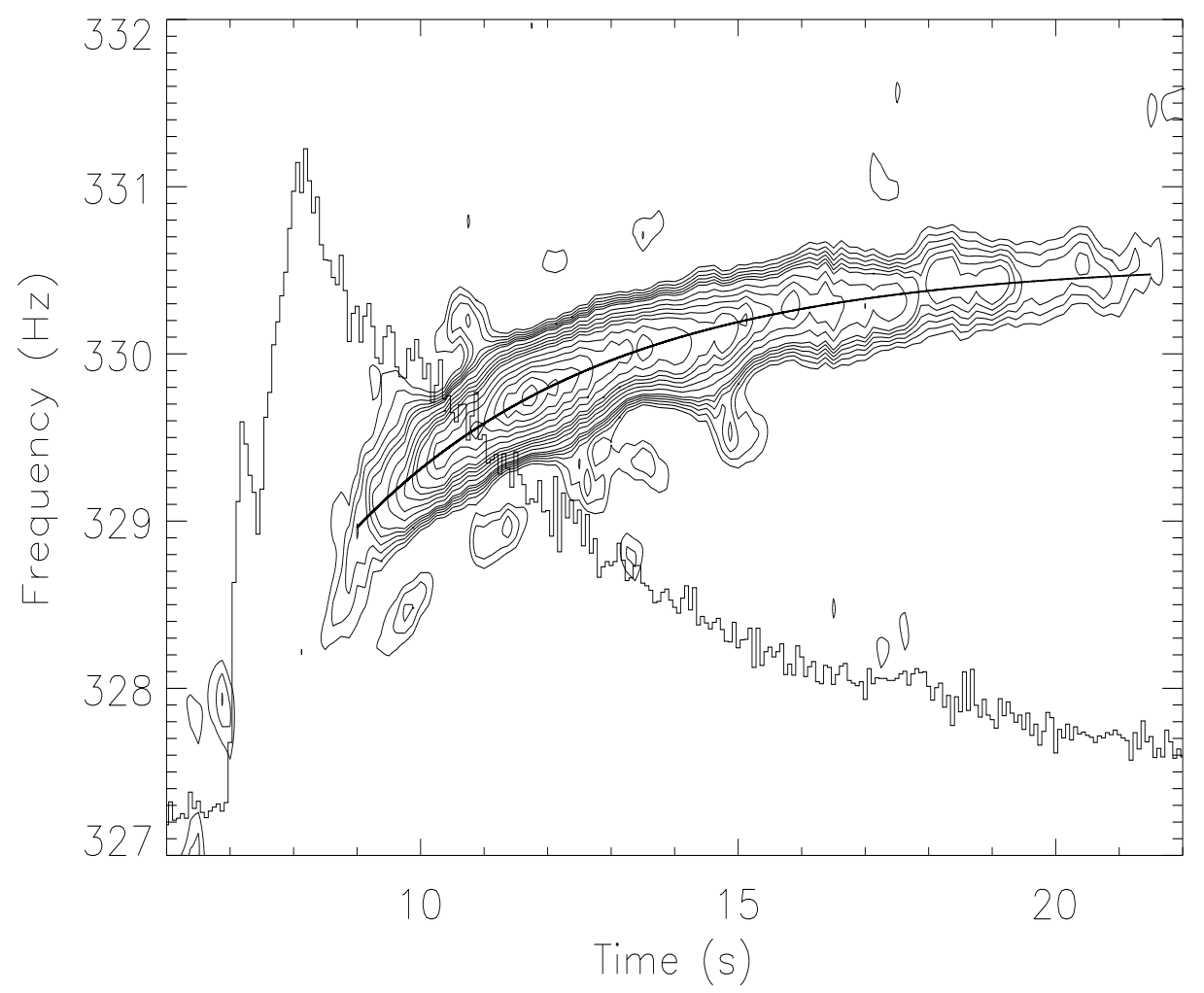

Fig. 3.9. An X-ray burst from $4 \mathrm{U} 1702-429$ observed with the PCA onboard RXTE. Shown are contours of constant power spectral density as a function of frequency and time. The solid curve shows the best fitting exponential model. The burst time profile is also shown (after Strohmayer \& Markwardt 1999).

of the thermonuclear burning layer because of the magnitude of the implied height change of $\sim 80 \mathrm{~m}$. Wijnands, Strohmayer \& Franco (2001) found a $\sim 5 \mathrm{~Hz}$ frequency shift in a burst from $4 \mathrm{U} 1658-298$ with a $567 \mathrm{~Hz}$ oscillation, which may also be uncomfortably large given current estimates of the expansion of the burning layers (Cumming et al. 2002).

\subsubsection{Long term stability of burst oscillation frequencies}

Studies of the frequency stability of burst oscillations over years provides constraints on the mechanism which sets the frequency. Strohmayer et al. (1998b) carried out one of the first studies of long term stability. They compared the asymptotic burst oscillation frequencies in bursts from $4 \mathrm{U} 1728-34$ and $4 \mathrm{U} 1636-53$. For bursts from $4 \mathrm{U} 1728-34$ spanning a 1.6 year epoch they found that the asymptotic pulsation period was the same to better than $1 \mu \mathrm{sec}$. This suggests a timescale to change the frequency longer than 23,000 years, and supports a mechanism like rotation with a high degree of intrinsic stability.

The accretion-induced rate of change of the neutron star spin frequency in a LMXB has a characteristic value of $1.8 \times 10^{-6} \mathrm{~Hz} \mathrm{yr}^{-1}$ for a canonical neutron star and 

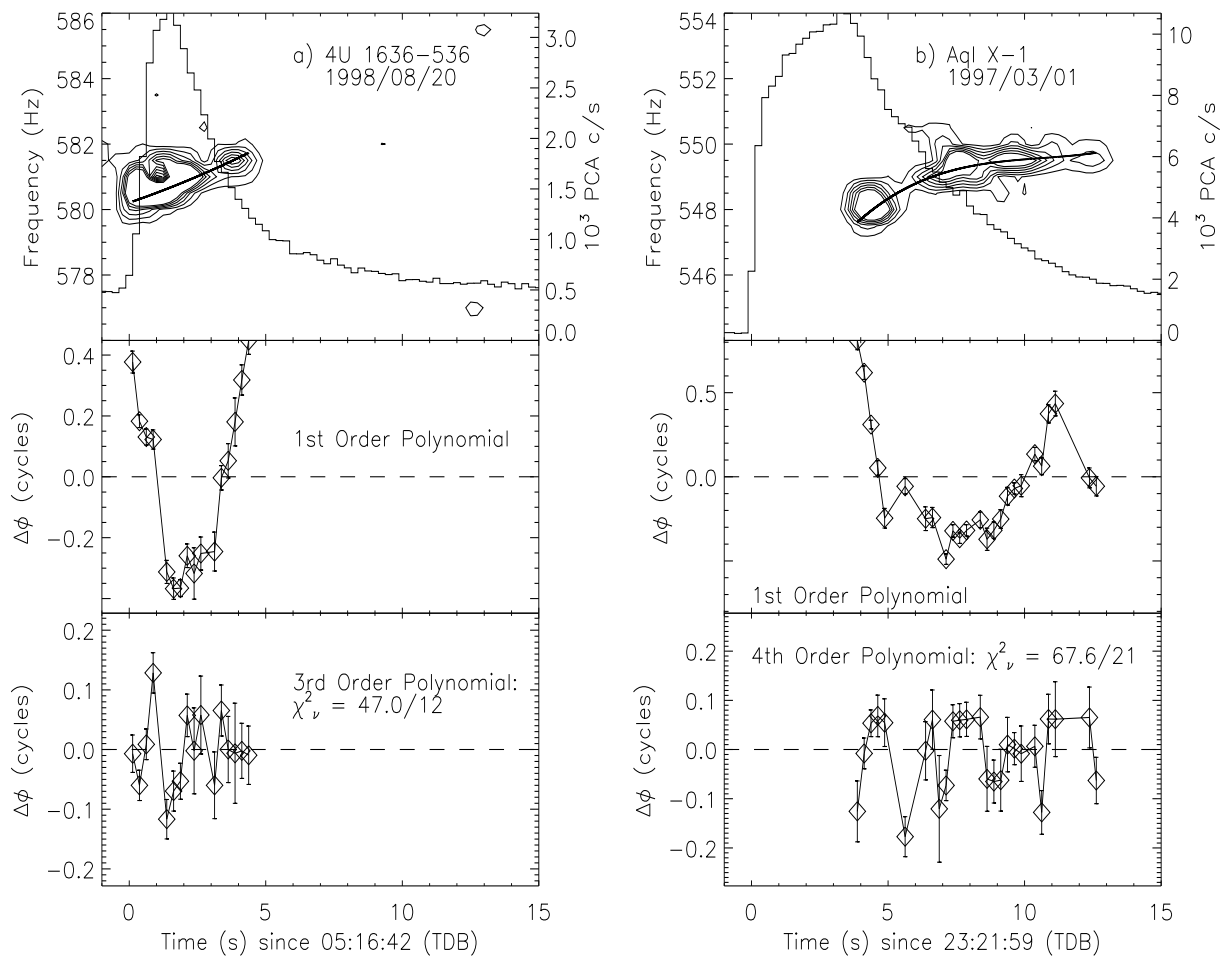

Fig. 3.10. Examples of two burst oscillations which show complex phase evolution. In both cases higher order polynomial phase evolution functions (3rd (left) and 4th (right) order) still leave significant residuals (after Muno et al. 2002).

typical mass accretion rates for X-ray burst sources. Over a year the accretion induced shift is much smaller than the apparent spin frequency changes which would be caused by the projected orbital motion of the neutron star. This led to the suggestion that, if burst oscillation frequencies were intrinsicaly stable enough, one might be able to extract the projected orbital velocity of the neutron star from a sample of bursts observed at different orbital phases. Recent studies, however, have confirmed that it will be much more difficult to extract neutron star velocity information from burst oscillation frequencies than initially hoped. For example, Giles et al. (2002) studied the burst oscillation frequencies of 26 bursts from the LMXB 4U 1636-53. The highest observed oscillation frequency of all bursts in their study are stable at the level of $2 \times 10^{-3}$, but are not correlated with orbital phase as expected for binary modulation.

In a related study Muno et al. (2002) examined the asymptotic burst oscillation frequencies of bursts from 8 different sources, including $4 \mathrm{U} 1636-53$. They quantify the dispersion in asymptotic frequencies using the standard deviation, $\sigma_{\nu}$, of the observed frequencies and find that $\sigma_{\nu} /\left\langle\nu_{\max }\right\rangle$ is typically $<1 \times 10^{-3}$. Figure 3.11 summarizes the distributions of asymptotic burst oscillation frequencies found 


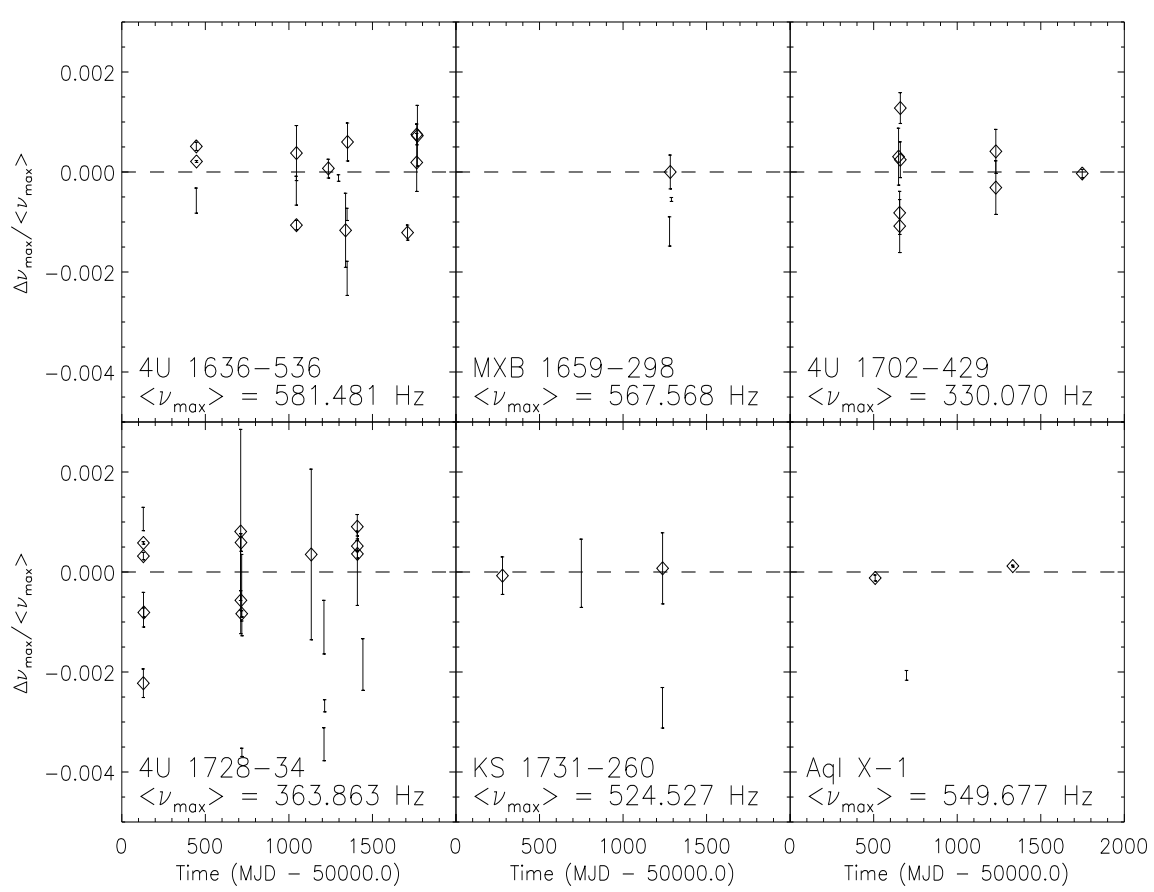

Fig. 3.11. The fractional deviations from the mean of maximum burst oscillation frequencies from 6 different burst sources. The data are shown as a function of time. The standard deviation, $\sigma_{\nu} /\left\langle\nu_{\max }\right\rangle$, is typically $7-10 \times 10^{-4}$ (after Muno et al. 2002).

in the Muno et al. (2002) study. These results indicate that the asymptotic burst oscillation frequencies are quite stable, but that there is more variation than can easily be accounted for by binary Doppler modulations alone. This requires that models for the burst oscillations must be able to account for an intrinsic fractional frequency variation perhaps as large as $\approx 5 \times 10^{-4}$.

One physical effect that approaches this magnitude is the change in the thickness of the burning layer from before the burst to after the burst due to the change in mean molecular weight (Cumming \& Bildsten 2000, see their figure 12). A complete burn of hydrogen via the rp-process can cause a fractional change in the rotation rate of $\approx 5 \times 10^{-4}$ (Cumming et al. 2002), so that varying levels of burning can lead to differing asymptotic frequencies at this level.

\subsubsection{Burst oscillations and the mass accretion rate}

Not all bursts from a given source have detectable oscillations. Recent work indicates that the mass accretion rate, $\dot{M}$, onto the neutron star has a strong influence on the strength, and therefore the detectability of burst oscillations. This is perhaps not too surprising, since $\dot{M}$ is known to influence other burst properties as well, and, as outlined earlier, is an important ingredient in any description of the nuclear 
burning physics. It is not unreasonable then to expect that the properties of burst oscillations might also depend importantly on $\dot{M}$.

Muno et al. (2000) carried out the first systematic study of burst oscillation properties and source spectral state. They studied bursts from the LMXB and atoll source KS 1731-260. The $524 \mathrm{~Hz}$ burst oscillations in this source were discovered by Smith, Morgan \& Bradt (1997). Muno et al. (2000) studied both the spectral and timing properties of bursts as a function of the position of the source in an X-ray color-color diagram (CD), and found that burst properties, including the presence or absence of burst oscillations, were strongly segregated in the CD. In particular, they found that only bursts which occurred when the source was located on the "banana branch" of the atoll pattern (i.e. at high inferred $\dot{M}$ ) produced detectable oscillations. These bursts also showed PRE, had the highest peak fluxes and had characteristically short durations (so called "fast" bursts). As discussed earlier these latter characteristics are an indication that helium is the primary fuel in such bursts (see $\S 3.2 .2$ ).

Subsequent studies of bursts from $4 \mathrm{U} 1728-34$ by van Straaten et al. (2001) and Franco (2001) confirmed some of the main results found by Muno et al. (2000), but new findings also complicated the picture. For example, both of these studies found a similar dependence of burst oscillations on source position in the CD. That is, bursts with oscillations are restricted to the banana branches at higher inferred $\dot{M}$. However, the properties of so called "fast" and "slow" bursts were not as nicely segregated in $4 \mathrm{U} 1728-34$ as for KS 1731-260. For example, the bursts at low inferred $\dot{M}$ (in the so called island state of the CD) from $4 \mathrm{U}$ 1728-34 all showed PRE whereas the corresponding bursts from KS 1731-260 do not. Moreover, some of the "fast" bursts from $4 \mathrm{U}$ 1728-34 which show strong burst oscillations show no evidence for PRE. Franco (2001) further characterized the bursts by computing a measure of integrated oscillation strength through the bursts. This integrated oscillation strength increased with inferred $\dot{M}$ on the CD (see Figure 3.12). A relationship between position in the $\mathrm{CD}$ and where during a burst oscillations are detected was also suggested. Bursts with oscillations detected only during the rising phase were found at the highest inferred $\dot{M}$, while bursts with oscillations only in the cooling (decay) phase were found at lower $\dot{M}$ (but still on the banana branch).

\subsubsection{Burst oscillations and photospheric radius expansion}

Soon after the discovery of the first burst oscillation sources a connection between the appearance of oscillations in bursts and PRE was apparent. Smith, Morgan \& Bradt (1997) found that oscillations in a PRE burst from KS 1731260 were first detected after photospheric touchdown. Strohmayer et al. (1997) found similar results for bursts with $589 \mathrm{~Hz}$ oscillations from a burster near the Galactic center. Muno et al. (2000) have explored the connection between PRE and oscillations in a large sample of bursts from several sources. They segregated burst oscillation sources into two classes, those with burst oscillation frequencies closer to $300 \mathrm{~Hz}$ (so called "slow" oscillators), and those with frequencies close to $600 \mathrm{~Hz}$ (the "fast" group), and found that those bursts with fast oscillations were almost always observed in bursts with PRE episodes, while bursts with slow oscillations only showed PRE about half the time. Muno et al. (2000) suggest that this distinction 


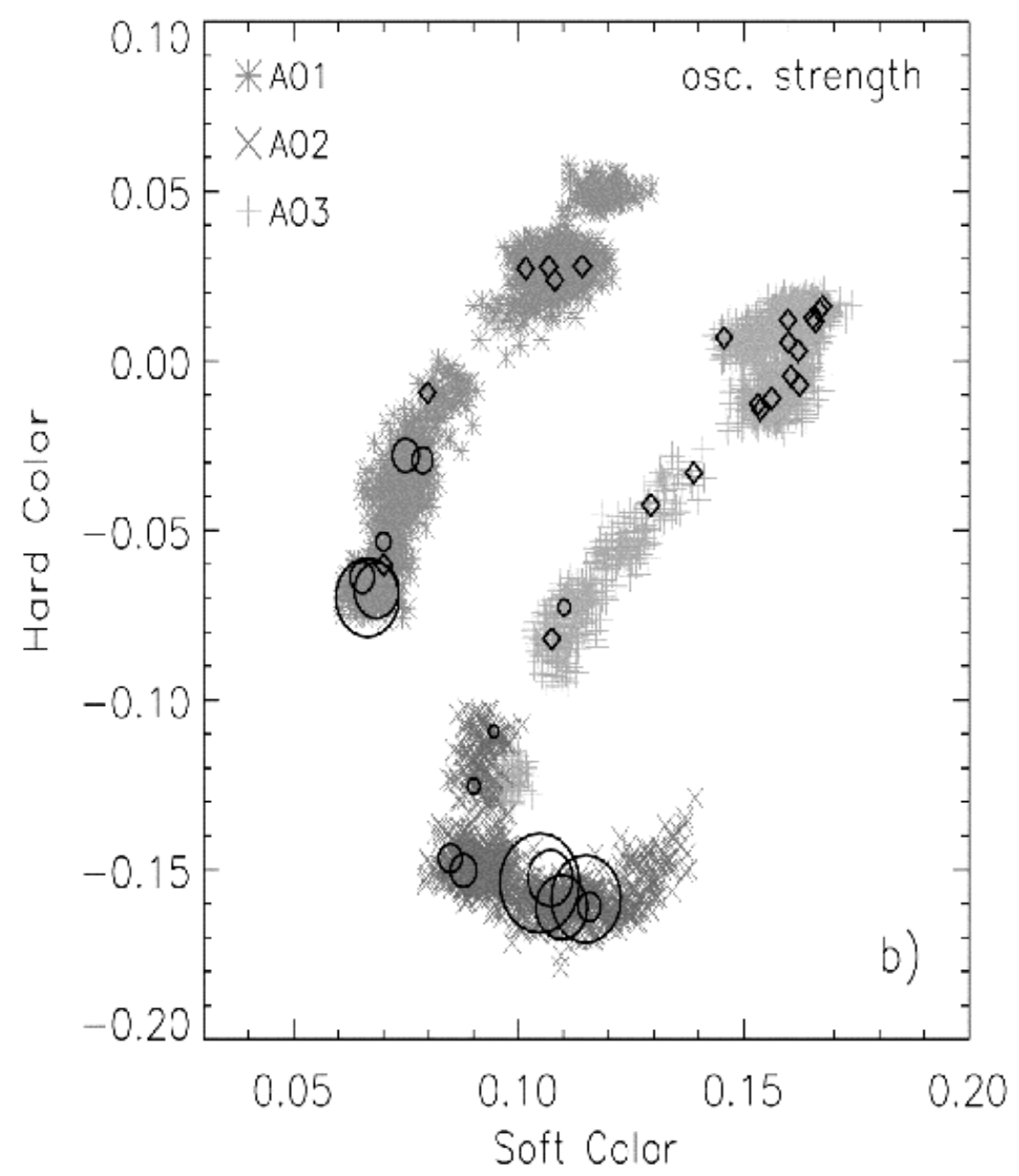

Fig. 3.12. X-ray color-color diagram for 4U 1728-34 showing a typical atoll track identifying, counterclockwise from upper right, the extreme island state (EIS), the island state (IS), the lower banana branch (LB), and the upper banana branch (UB). The locations of bursts both with (circles) and without (diamonds) oscillations are shown. For bursts with oscillations, the size of the circle is proportional to the integrated strength of the oscillations (after Franco 2001).

between the fast and slow sources cannot be an observational selection effect. They argue that the distinction could result if burst properties vary differently with $\dot{M}$ in the fast and slow sources. Exactly how this difference comes about and how it is related to the oscillation (or spin) is not yet understood.

\subsubsection{Harmonics, subharmonics and sidebands}

The pulse shapes of burst oscillations are highly sinusoidal. Strohmayer \& Markwardt (1999) found no evidence for harmonics after coherently summing oscillation signals from $4 \mathrm{U} 1728-34$ and $4 \mathrm{U}$ 1702-429. They placed limits on the 
ratio of pulsed amplitude at the fundamental to that at the first harmonic of $\approx 24$ and 15 in these sources, respectively. Muno et al. (2000), found only weak $(2 \sigma)$ evidence of a signal at the first harmonic of the $524 \mathrm{~Hz}$ frequency in bursts from KS 1731-260. Recently, Muno, Özel \& Chakrabarty (2002) have explored the amplitude evolution and harmonic content of cooling phase burst oscillations in eight different sources. They find mean amplitudes (rms) of about $5 \%$, and during a typical burst the amplitude can vary in a manner which is uncorrelated with the burst flux. They did not detect any harmonic, nor subharmonic signals, and placed upper limits on the fractional amplitudes at integer and half-integer harmonics of less than $5 \%$ and $10 \%$ of the amplitude of the strongest observed signal, respectively. Comparison of these results to theoretical models with one or two circular, antipodal hot spots suggests that if a single spot is present it must lie near the rotational pole or cover a substantial fraction $(\approx 1 / 2)$ of the neutron star in order to be consistent with the limits on harmonic signals. If antipodal spots are present, then the implications are that the spots must lie close to the rotational equator.

Chakrabarty (2002) has found evidence for sidebands separated by $30-50 \mathrm{~Hz}$ from the burst oscillation frequency in a few sources. These sidebands do not yet fit cleanly within the context of current models, however, Spitkovsky et al. (2002) have argued that zonal flows associated with burst heating and rapid spin of the neutron star may produce a modulation pattern as well as sidebands (see §3.4.11).

In many LMXBs a pair of $\mathrm{kHz}$ QPOs are observed (see the review by van der Klis in Chapter 2). In six sources the frequency difference between the $\mathrm{kHz}$ QPO is close to the observed burst oscillation frequency or one half of the frequency. This closeness of the burst oscillation frequency, and the frequency difference of the $\mathrm{kHz}$ QPOs has motivated the "beat frequency" models of kHz QPOs (see Strohmayer et al. 1996; Miller, Lamb \& Psaltis 1998; Lamb \& Miller 2001). In three sources the QPO difference frequency is close to half the burst oscillation frequency. This might be possible if a pair of antipodal hot spots on the neutron star produces the burst oscillation. Based on these considerations, Miller (1999) searched for a subharmonic of the $581 \mathrm{~Hz}$ burst oscillation in $4 \mathrm{U} 1636-53$, and claimed detection of a $290 \mathrm{~Hz}$ signal at a $4 \times 10^{-5}$ significance level. Extensive efforts to detect such a signal again have, however, been unsuccessful (see Strohmayer 2001 for a discussion).

\subsubsection{Burst oscillations as probes of neutron stars}

Modelling of burst oscillations holds great promise as a new tool for probing the structure of neutron stars and their environs. The emission and propagation of photons from the surfaces of rapidly rotating neutron stars are strongly influenced by General relativistic effects. Gravitational light deflection suppresses the modulation amplitude and reduces the harmonic content of pulses produced by rotational modulation of a hot spot on a rotating neutron star (see Pechenik, Ftaclas \& Cohen 1983). The strength of light deflection is a function of the compactness, $m / r \equiv G M / c^{2} R$. More compact stars produce greater deflections and therefore weaker spin modulations (see Strohmayer et al. 1998a; Miller \& Lamb 1999). Relativistic motion of the hot spot creates asymmetry and sharpening of the pulse profile, increasing the harmonic content (Chen \& Shaham; Miller \& Lamb 1999; Braje, Romani \& Rauch 2000; Weinberg, Miller \& Lamb 2001). Such motion also introduces a pulse phase 
dependent Doppler shift in the X-ray spectrum. The magnitude of these effects are directly proportional to the surface velocity, which is a function of the unknown stellar radius and the known spin frequency. Ford (1999) has analysed data during a burst from Aql X-1 and finds that the softer photons lag higher energy photons in a manner which is qualitatively similar to that expected from a rotating hot spot. Fox (2001), however, found that the sense of the phase lags in this burst switched, with hard lags preceding the soft lags found by Ford et al. (1999). This suggests that photon scattering may play an important role in addition to Doppler beaming.

Several studies have been undertaken to constrain neutron star properties based on modelling of burst oscillations. Miller \& Lamb (1998) have investigated the amplitude of rotational modulation pulsations as well as harmonic content assuming emission from a point-like hot spot. They also showed that knowledge of the angular and spectral dependence of the emissivity from the neutron star surface can have important consequences for the derived constraints. Nath, Strohmayer \& Swank (2001) have modelled bolometric pulse profiles observed during the rising phase of bursts from $4 \mathrm{U}$ 1636-53. They fit the pulse profiles with a rotating, expanding hot spot model that includes light deflection in the Schwarzschild spacetime. They find that the inferred constraints depend very sensitively on whether or not two spots are present. Much more restrictive compactness constraints can be achieved if two spots are present. The main reason being that large amplitudes are much more difficult to achieve with two spots than one. Assuming two hot spots they find a lower limit to the compactness of $m / r<0.163$ at $90 \%$ confidence. This requires a relatively stiff equation of state for the neutron star interior and disagrees with the recently measured value of $m / r=0.23$ from Cottam et al. (2002). If one hot spot is assumed, then the constraint is consistent with the Cottam et al. (2002) measurement. Weinberg, Miller \& Lamb (2001) have explored the oscillation waveforms and amplitudes produced by rotating neutron stars with single and antipodal hot spots of varying size. They include photon propagation in the Schwarzschild spacetime and consider the effects of relativistic aberration and Doppler shifts induced by the rotational motion of the neutron star surface. They concluded that pulse profile fitting could be a powerful tool in constraining neutron star properties.

\subsubsection{Spin modulation: implications for spin in LMXBs}

The discovery of burst oscillations provided the first strong observational evidence for millisecond rotation periods in accreting neutron star LMXB's. Since then three bonafide accreting millisecond pulsars have been discovered; SAX J1808.4-3658 (Wijnands \& van der Klis 1998; Chakrabarty \& Morgan 1998), XTE J1751-305 (Markwardt et al. 2002), and XTE J0929-314 (Galloway et al. 2002), with spin frequencies of $401 \mathrm{~Hz}, 435 \mathrm{~Hz}$, and $185 \mathrm{~Hz}$, respectively. These pulsars all have extremely low mass companions, with $M_{c} \approx 0.01 M_{\odot}$ (for XTE J1751-305 and XTE J0929-314, Bildsten 2002), and they have firmly established the link between millisecond radio pulsars and accreting LMXBs. However, until very recently no source had shown both persistent pulsations and burst oscillations at the same frequency, with the exception of a $3 \sigma$ detection with the WFC of an oscillation at $401 \mathrm{~Hz}$ during a burst from SAX J1808.4-3658 (in 't Zand et al. 2001).

A few weeks before having to finalize our review, a new outburst of the accreting 
millisecond pulsar SAX J1808.4-3658 was discovered with the RXTE/ASM (Markwardt, Miller \& Wijnands 2002). Extensive RXTE observations of the outburst have so far detected four thermonuclear bursts, all of which are PRE bursts. Each burst also shows oscillations, with pulsations detected during the rise and after "touchdown" of the photosphere, as is seen in many other burst oscillation sources which do not show pulsations in their persistent emission. The oscillations in the bursts from SAX J1808.4-3658 are clearly distinct from the coherent pulsations in the persistent flux because the oscillation amplitude is larger. Oscillations seen in the decaying tails were at the spin frequency (Wijnands et al. 2002). These recent findings, as well as the discovery of coherent pulsations during a superburst from $4 \mathrm{U} 1636-53$ (see Strohmayer \& Markwardt 2002; $§ 3.5 .4$ below) conclusively establish that burst oscillations result from spin modulation of the X-ray burst flux.

\subsubsection{Theoretical implications of burst oscillations}

The spin modulation mechanism requires a slow-moving, non-uniform brightness pattern on the neutron star surface. At burst onset the pattern is most likely a localized "hot spot", whereas we are still mostly in the dark as to the origin of oscillations long after the burning has started. What all of the observations have made clear is that we can no longer persist with spherically symmetric modelling. In many ways, the question we now need to answer is: "What breaks the symmetry?" as prior to and after the bursts, there is no indication of azimuthal variations on the stellar surface.

The initial work on the spreading of a locally ignited (i.e. symmetry broken by hand) "hot spot" away from the ignition site were focused on the laminar or convective combustion viewpoint (e.g. Fryxell \& Woosley 1982; Bildsten 1995) and neglected the physics of the atmospheric response to transverse pressure gradients. Such an approximation is only appropriate if the matter is very degenerate. In this limit, the observed short rise times can only be explained with convective velocities. However, for most observed bursts the degeneracy is only partial during the flash. In this case, an additional velocity becomes important, which is the shallow water wave speed, $V_{s w} \approx(g h)^{1 / 2}$, where $g$ is the surface gravity and $h \approx 10$ meters is the thickness of the burning layer. This speed is $\approx 4000 \mathrm{~km} / \mathrm{sec}$, so that, in the absence of rotation, any transverse pressure disturbances create wave-like disturbances with periods as short as 5-10 milliseconds (e.g. Livio \& Bath 1982; McDermott \& Taam 1987; Bildsten \& Cutler 1995; Bildsten \& Cumming 1998). However, these periods are longer than the neutron star rotation period, in which case the Coriolis force must be taken into account (see Bildsten et al. 1996; Strohmayer \& Lee 1996 for the rotational modification of modes) when considering the nature of the modes.

Spitkovsky et al. (2002) showed that this same interplay between the shallowwave speed and the Coriolis force is relevant to the propagation speed of localized burning on a rotating star. In their groundbreaking calculation, they found that the width of the burning front at the leading edge of the hot spot was the Rossby adjustment radius $\sim V_{s w} / \Omega$, and the resulting speed of the front is $V_{f} \sim V_{s w} /\left(\Omega t_{n}\right) \sim$ $2-10 \mathrm{~km} \mathrm{~s}^{-1}$, where $t_{n} \sim 0.1-1$ second is the time to burn the fuel (for example, in a helium-rich flash). The spreading time for the whole star is then 0.1 to a few seconds, in the observed range. The dependence on the rotation rate, $\Omega$, would be 


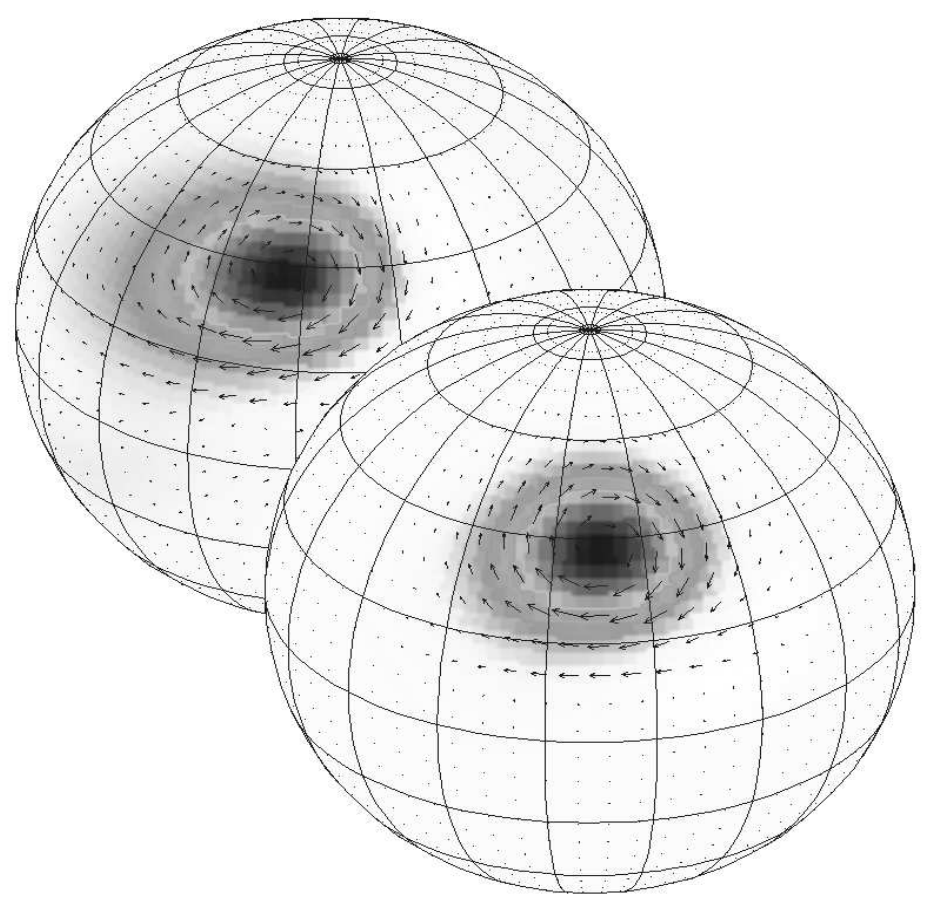

Fig. 3.13. Initial evolution of a burning hot spot ignited off the equator as seen in a frame rotating with the neutron star. Velocity vectors show the circulation of the fluid induced by the Coriolis forces. The hot spot expands due to burning and drifts west-southwest because of the latitude dependence of the Coriolis force (after Spitkovsky, Levin \& Ushomirsky 2002).

nice to test, but the current range of measured rotation rates in bursters is likely too small to allow for it.

Spitkovsky et al. (2002) also investigated the dynamics of the nuclear ignition on a rotating star and argued that ignition will tend to occur in the equatorial region and propagate to the poles (see Figure 3.13). The combination of radial uplift and horizontal flows they found may also be able to explain the observed frequency drifts. This initial progress is remarkable, but much remains to be done, including an implementation of more realistic burning during the propagation and an improved understanding of where the accreted fuel resides and where/how ignition really starts in a three-dimensional star. The standard ignition condition is for a spherically symmetric perturbation of a spherically symmetric model and most likely both of those approximations need to be dropped.

In the burst tails the oscillation might conceivably be produced by a mode (e.g. McDermott \& Taam 1987) or perhaps is generated dynamically by the interaction of burst heating and cooling with the rapid spin of the star (see Spitkovsky et al. 2002). 
Indeed, the pure harmonic content points to an azimuthal perturbation $\propto \exp (i m \phi)$. However, any such model must explain both the $\approx 1 \%$ frequency drift and the longterm stability and none convincingly have.

Without specifically addressing the mode question, Cumming \& Bildsten (2000) explored in detail the radial uplift mechanism outlined by Strohmayer et al. (1997) to explain the frequency drifts. In the context of presuming that the burning layer became disconnected from the underlying material but itself rigidly rotated, they concluded that this process could explain most of the drifts. Heyl (2000) claimed that properly including general relativistic effects would allow for strong constraints on the NS radius, but soon thereafter Abramowicz, Kluzniak \& Lasota (2001) and Cumming et al. (2002) found an error in Heyl's formulation that strongly reduced the impact of general relativity. However, during this reevaluation, Cumming et al. (2002) found that they had overestimated the radial uplift drift by about a factor of two. This result, combined with the observations of much larger drifts in several sources (Galloway et al. 2001, Wijnands et al. 2002) now makes it appear that radial uplift is insufficient to account for all the observed drift if the whole burning layer is rigidly rotating. One possible way out from this conundrum is to allow some fluid elements in the burning layer to keep their initial angular momentum (i.e. not demand rigid rotation of the whole burning layer). This easily produces much larger frequency shifts for the outermost layers (Cumming and Bildsten 2000), but the origin of such a coherent signal in the context of the rapid internal differential rotation is then a new puzzle to solve. These uncertainties will likely remain with us until the origin of the asymmetry is resolved.

The strength of the large-scale dipole field that threads the burning layers on the neutron star is unknown. The lack of persistent pulsations during accretion leads to the limit of $B<10^{8-9} \mathrm{G}$ by presuming that the spherical magnetospheric radius is inside the neutron star. However, $B$ might need to be even lower than this to ensure azimuthal symmetry. For example, it is easy to imagine that a permanent asymmetry could result even if the material arrives on the equator, but encounters an ordered field as it tries to spread away as the photospheric pressure is only $\approx 10^{15} \mathrm{erg} \mathrm{cm}^{-3}$. In addition, during the bursts, if the frequency drift that is observed is due to vertical shear from the radially expanded burning layers, an initially poloidal field as weak as $10^{6} \mathrm{G}$ could become dynamically important as it is wound up by differential rotation (Cumming \& Bildsten 2000).

\subsection{Superbursts: a new burning regime}

Since the advent of BeppoSAX and RXTE in 1996, the X-ray sky has been monitored with unprecedented sensitivity and frequency. This capability has opened up the discovery space for burst events with long (years) recurrence times, which were apparently missed by previous missions. Cornelisse et al. (2000) reported the first superburst discovery (from BeppoSAX) in the familiar Type I burster 4U 173544. This was rapidly followed by reports of six more long X-ray flares lasting 3 5 hours from five previously known X-ray bursters; 4U 1820-30 (Strohmayer 2000, Strohmayer \& Brown 2001); KS 1731-260 (Kuulkers et al. 2002c); 4U 1636-53 (Wijnands 2001; Strohmayer \& Markwardt 2002); Ser X-1 (Cornelisse et al. 2002c); and GX $3+1$ (Kuulkers 2002). One of the sources, $4 \mathrm{U}$ 1636-53, produced two bursts 

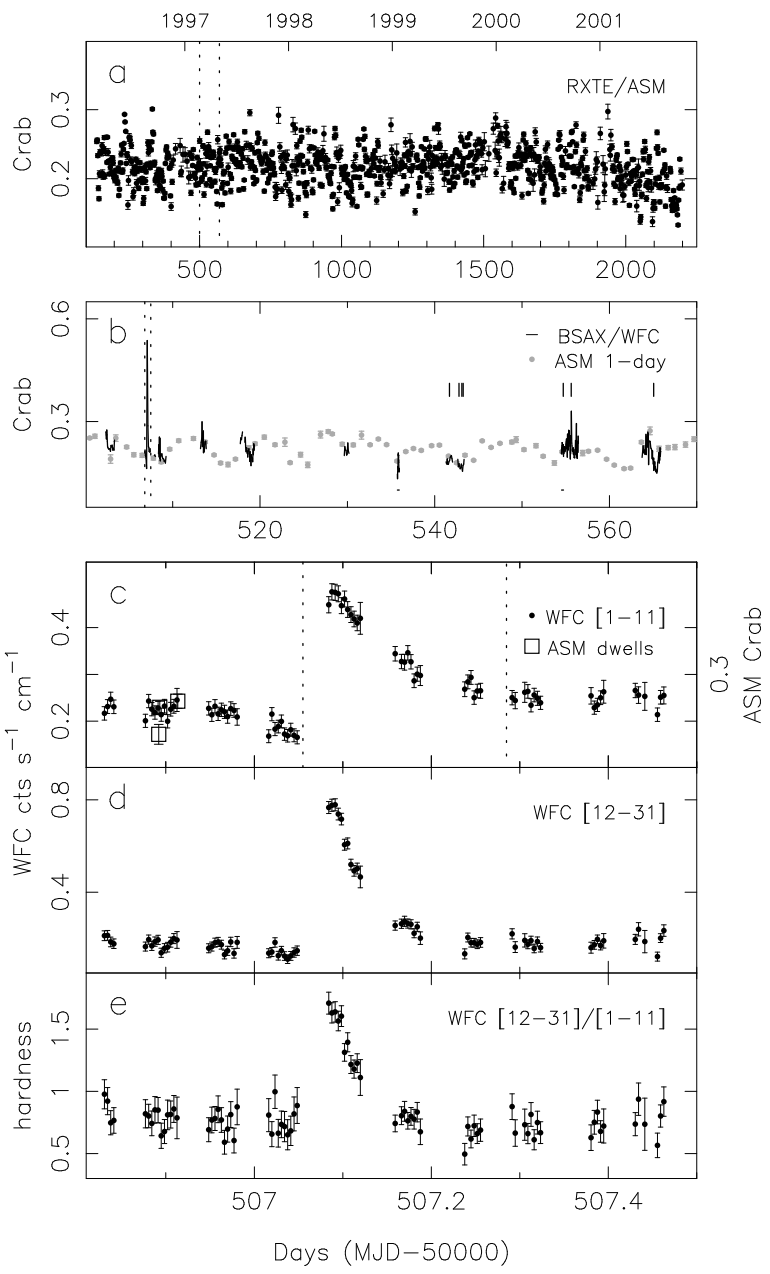

Fig. 3.14. The superburst from Ser X-1 observed with the WFC on BeppoSAX. Note the persistent offset in the flux after the superburst in the $2-5 \mathrm{keV}$ band (c). The hardness ratio (e) drops through the burst, indicative of cooling of the neutron star surface (after Cornelisse et al. 2002c).

separated by 4.7 years (Wijnands 2001). Two of the events, one from $4 \mathrm{U} 1636-53$, and the other from $4 \mathrm{U} 1820-30$ were observed with the large area PCA onboard RXTE (Figures 3.14 and 3.15 show superbursts seen with the BeppoSAX/WFC and RXTE/PCA, respectively).

Table 3.2 (after Kuulkers et al. 2002c) summarizes the important properties of the superbursts observed to date. These flares show all the hallmarks of thermonuclear bursts; they have thermal spectra which soften with time, they show more or less smooth exponential-like decays, and some show PRE episodes. The fundamental distinctions between these events and standard type I X-ray bursts are their long durations, larger fluences and long recurrence times (ie. they are rare). Indeed, these events are typically 1,000 times longer and more energetic than standard X- 
ray bursts, because of this, they have come to be referred to as "superbursts," and we will continue to use this appellation. Although the sample size is still relatively small, the systems which have produced superbursts have typical accretion rates in the range $\approx 0.1-0.3$ of $\dot{M}_{E d d}$ (Wijnands 2001; Kuulkers et al. 2002c). As we describe in more detail shortly, this is an important constraint on theoretical models.

The durations and energetics of superbursts suggest that they result from thermonuclear flashes occurring in fuel layers at much greater depth than for typical $\mathrm{X}$-ray bursts. For example, if the nuclear energy release is $0.3 \mathrm{MeV}$ per accreted nucleon, the accumulated mass required to power a $10^{42} \mathrm{erg}$ superburst is $3.5 \times 10^{24} \mathrm{~g}$, giving a recurrence time of $\approx 2$ years for an accretion rate of $\dot{M} \approx 10^{-9} M_{\odot} \mathrm{yr}^{-1}$. In the remainder of this section we will summarize the current state of knowledge of superbursts and explore what new insights they are giving us about nuclear burning on neutron stars.

\subsubsection{Time profiles and spectra}

The most distinctive characteristic of superbursts are their long durations. The time profiles of most superbursts have been characterized with an exponential decay time, and these values range from about 1 - 3 hours (see Kuulkers et al. 2002c). The longest event seen to date was the superburst from KS 1731-260, which lasted for almost half a day (Kuulkers et al. 2002c). Their time profiles are generally smooth, with a fast rise and exponential decay, however, not all superbursts have been observed with comparable sensitivity and temporal resolution, so comparisons must be made with some caution. For example, the superburst from $4 \mathrm{U} 1820$ 30 showed substantial variability during portions of the decay phase (Strohmayer \& Brown 2002). Both superbursts which were observed with the large area PCA show "precursor" events just prior to the start of the superburst (see Strohmayer \& Brown 2002; Strohmayer \& Markwardt 2002; Figure 3.15). These precursors look more or less like standard type I bursts from the respective objects. There is also some evidence for a precursor in the burst from KS 1731-260 observed with the BeppoSAX/WFC (Kuulkers et al. 2002c).

Based on the available data, it appears that such precursors are a common feature of superbursts. If the superbursts result from energy release at great depths, then it seems plausible that the superburst flux could trigger a flash in the $\mathrm{H} / \mathrm{He}$ layers above it as it diffuses outward. There has been some speculation that the precursor may act as a trigger for the superburst, but given the much longer radiative diffusion time at the depth where the superbursts are likely triggered, this seems unlikely (see Strohmayer \& Brown 2002).

The X-ray spectra of superbursts are well described by thermal emission, with peak blackbody temperatures in the range from $2-3 \mathrm{keV}$, quite typical for thermonuclear bursts (Cornelisse et al. 2000). Interestingly, most of the observed superbursts have peak fluxes which are sub-Eddington, only the superburst from the pure helium accretor $4 \mathrm{U}$ 1820-30, had a peak flux consistent with the Eddington limit.

Strohmayer \& Brown (2002) found discrete components in the spectra of the superburst from $4 \mathrm{U}$ 1820-30; a broad emission line centered near $6 \mathrm{keV}$ and an accompanying absorption edge between 8 and $9 \mathrm{keV}$ (see Figure 3.5). They suggested that these features can be explained by reflection of the burst flux from the inner accretion 
Table 3.2. Superburst sources and properties (after Kuulkers et al. 2002c)

\begin{tabular}{|c|c|c|c|c|c|c|}
\hline $\begin{array}{l}\text { Source } \\
\text { detector }^{a}\end{array}$ & $\begin{array}{c}4 \mathrm{U} 1820-30 \\
\text { PCA }\end{array}$ & $\begin{array}{c}4 \mathrm{U} 1735-44 \\
\text { WFC }\end{array}$ & $\begin{array}{c}\text { KS } 1731-260 \\
\text { WFC, ASM }\end{array}$ & $\begin{array}{l}4 \mathrm{U} 1636-53 \\
\text { PCA, ASM }\end{array}$ & $\begin{array}{c}\text { Ser X-1 } \\
\text { WFC }\end{array}$ & $\begin{array}{c}\text { GX } 3+1 \\
\text { ASM }\end{array}$ \\
\hline Duration $^{b}$ & 3 & 7 & 12 & $>2-3$ & $\approx 4$ & $>3.3$ \\
\hline Precursor? & yes & $?$ & yes & yes & $?$ & $?$ \\
\hline$\tau_{\exp }(\mathrm{hr})$ & $\approx 1$ & $1.4 \pm 0.1$ & $2.7 \pm 0.1$ & $\begin{array}{l}1.5 \pm 0.1 \\
3.1 \pm 0.5\end{array}$ & $1.2 \pm 0.1$ & $1.6 \pm 0.2$ \\
\hline$L_{\text {pers }}{ }^{c}$ & $\approx 0.1$ & $\approx 0.25$ & $\approx 0.1$ & $\approx 0.1$ & $\approx 0.2$ & $\approx 0.2$ \\
\hline$k T_{\max }{ }^{d}$ & $\approx 3$ & $\approx 2.6$ & $\approx 2.4$ & $?$ & $\approx 2.6$ & $\sim 2$ \\
\hline$L_{\text {peak }}^{e}$ & 3.4 & 1.5 & 1.4 & 1.2 & 1.6 & 0.8 \\
\hline$E_{b}^{f}$ & $>1.4$ & $>0.5$ & $\approx 1$ & $0.5-1$ & $\approx 0.8$ & $>0.6$ \\
\hline$t_{\text {quench }^{g}}{ }^{g}$ & $?$ & $>7.5$ & $>35$ & $?$ & $\sim 34$ & $?$ \\
\hline References $^{h}$ & S00, SB02 & $\mathrm{C} 00$ & $\mathrm{~K} 02 \mathrm{c}$ & W01, SM02 & $\mathrm{C} 02$ & K02 \\
\hline
\end{tabular}

${ }^{a}$ Instruments which observed the superburst.

${ }^{b}$ In hours.

${ }^{c}$ Persistent luminosity prior to superburst, in terms of the Eddington luminosity.

${ }^{d}$ In $\mathrm{keV}$.

e In units of $10^{38} \mathrm{ergs} \mathrm{s}^{-1}$.

$f$ in units of $10^{42}$ ergs.

$g$ Time following superburst with no normal burst activity, in days.

${ }^{h}$ S00 (Strohmayer 2000); SB02 (Strohmayer \& Brown 2002); C00 (Cornelisse et al. 2000); K02c (Kuulkers et al. 2002c); W01 (Wijnands 2001); SM02 (Strohmayer \& Markwardt 2002); C02 (Cornelisse et al. 2002c); K02 (Kuulkers 2002).

disk around the neutron star. Day \& Done (1991) had predicted that such features might be detectable in spectra of bursts. The energy and width of the dominant Fe K $\alpha$ fluorescence line as well as the energy of the edge can be used as important diagnostics of the ionization state of the disk (see for example, Ross, Fabian \& Young 1999; Nayakshin \& Kallman 2001). An origin of the features in a burst-driven wind is also possible. Because superbursts last about a 1,000 times longer than normal bursts and can give very high signal to noise spectra, they would allow much more sensitive searches for discrete spectral lines from neutron star surfaces. Superbursts would therefore make tempting targets for rapidly triggered observations with sensitive, high spectral resolution observatories. The soon to be launched Swift mission may be able to provide the necessary triggering capability.

\subsubsection{Superburst energetics}

The available X-ray spectroscopy on superbursts provides estimates of the total X-ray energy liberated by the events. For the superburst from $4 \mathrm{U} 1820-30 \mathrm{a}$ lower limit to the energy fluence was $1.5 \times 10^{42} \mathrm{ergs}$, assuming a distance of $6.6 \mathrm{kpc}$ (Strohmayer \& Brown 2002). It is extremely unlikely that unstable helium burning could provide such a large fluence. This could only occur at very low atmospheric temperatures and thus at low mass accretion rates inconsistent with the persistent X-ray flux observed from 4U 1820-30 (Fryxell \& Woosley 1982; Zingale et al. 2001). Similar fluence limits have been derived for the other superbursts as well (see Kuulkers et al. 2002c). The total energy observed in X-rays, combined with the decay 
timescales of superbursts strongly argues for a fuel source located at depths below the column density where helium flashes are triggered.

\subsubsection{Quenching of normal burst activity}

There are strong indications from several of the superburst sources that the occurrence of a superburst has a profound influence on the thermal state of the accreted "ocean" on the neutron star. In particular the occurrence of normal (short duration) X-ray bursts appears to be suppressed for some time following superbursts. For example, Kuulkers et al. (2002c) and Cornelisse et al. (2002c) found that normal bursting ceased for about 35 days following the superbursts from KS 1731-260 and Ser X-1, respectively (see Figure 3.14, where the tick marks in panel b denote the positions of normal bursts). There are also indications that normal bursting was suppressed for at least a week following the superburst from 4U 1735-44 (Cornelisse et al. 2000). A likely explanation of this suppression is that flux from the deep parts of the neutron star ocean remains high enough to quench the Type I bursting activity (Cumming \& Bildsten 2001).

\subsubsection{Millisecond pulsations during a superburst: $4 U$ 1636-53}

Burst oscillations at $\approx 582 \mathrm{~Hz}$ have been observed in many bursts from the LMXB 4U 1636-53 (see Table 3.1; Zhang et al. 1997: Giles et al. 2002). Strohmayer \& Markwardt (2002) recently discovered coherent pulsations at this frequency during a portion of the February 22, 2001 (UT) superburst from this source. They were detected during an $\approx 800 \mathrm{~s}$ interval spanning the flux maximum of the superburst. The average pulsation amplitude was $1 \%$, which is smaller than the amplitudes of oscillations observed from standard bursts. The pulse trains observed during the superburst are much longer than the typical, $10 \mathrm{~s}$ long pulse trains observed in normal bursts. The pulsation frequency was found to increase in a monotonic fashion by less than a part in $10^{4}$ (see Figure 3.16). This is a much smaller frequency drift than commonly seen in burst oscillations from standard bursts (see §3.4.4). The form of the frequency evolution appears consistent with Doppler modulation caused by the known orbital motion of the neutron star around the center of mass of the binary. Strohmayer \& Markwardt (2002) showed that a circular orbit model fits the observed frequency evolution well. The best phase evolution model is consistent with a coherent pulsation during the observation interval, and gives a limit on the coherence $Q \equiv \nu_{0} / \Delta_{\nu_{0}}>4.5 \times 10^{5}$. The orbital fits indicate that the projected neutron star velocity lies between 90 and $175 \mathrm{~km} \mathrm{~s}^{-1}$. The brevity of the observed pulse train with respect to the orbital period of $3.8 \mathrm{hr}$ does not allow for more precise constraints. The fact that the coherent pulsation frequency during the superburst is within $\approx 1 \mathrm{~Hz}$ of all the measured asymptotic burst oscillation frequencies for $4 \mathrm{U}$ 1636-53 indicates that the frequencies are set by the spin of the neutron star.

\subsubsection{Theory of Superbursts: Ashes to Ashes}

Unstable burning of a pure carbon layer (Woosley \& Taam 1976; Taam \& Picklum 1978; Brown \& Bildsten 1998) has been proposed as an explanation for the superburst from the pure helium accretor 4U 1820-30 (Strohmayer \& Brown 2002). In this scenario, the accumulated mass of carbon at ignition is $10^{26}-10^{27} \mathrm{~g}$, 


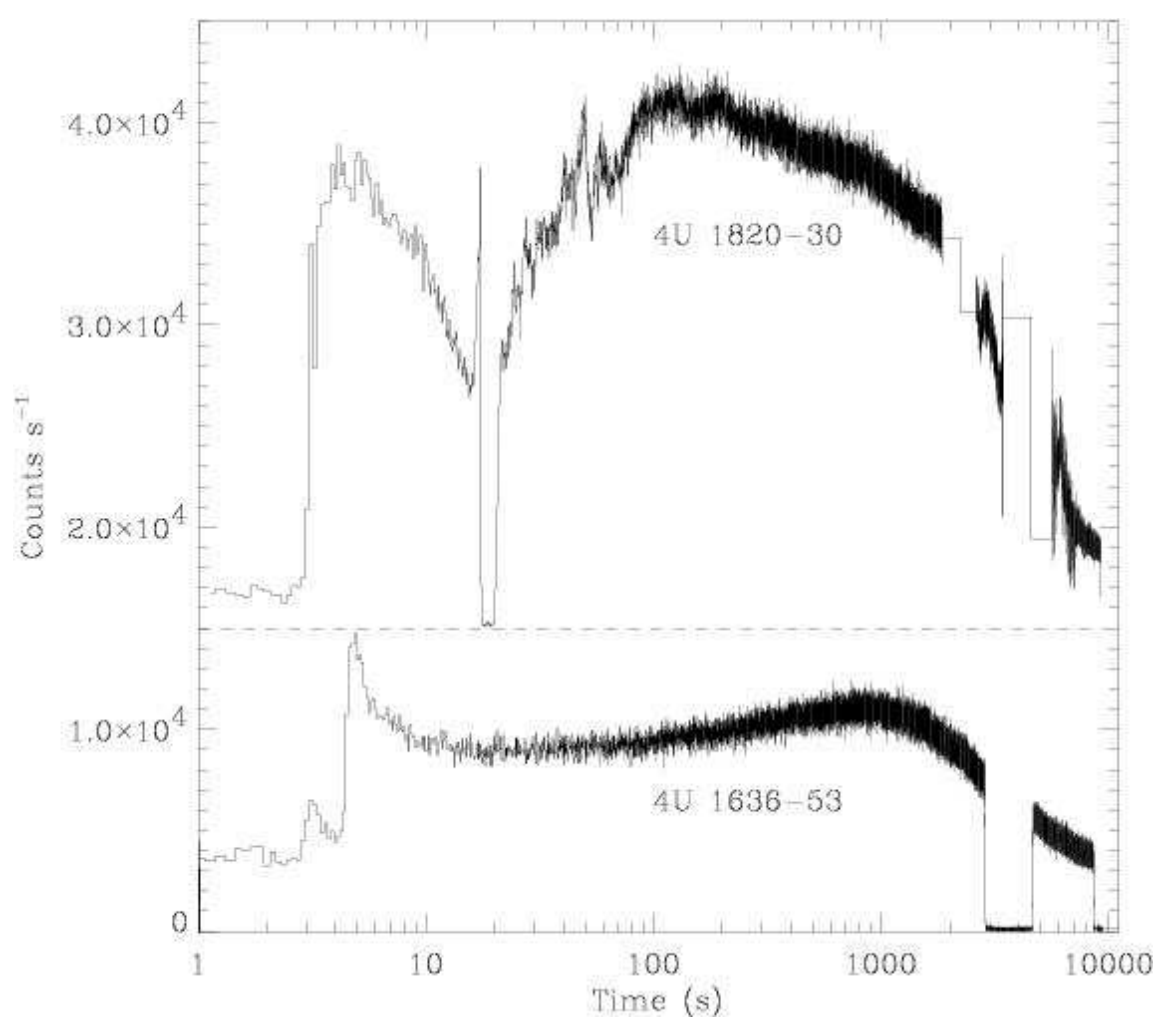

Fig. 3.15. Two superbursts observed with the RXTE/PCA. Shown are the $2-30 \mathrm{keV}$ count rate histories observed in the PCA. Note the shorter precursor events prior to the superbursts. The event from $4 \mathrm{U} 1820-30$ has been displaced vertically for clarity. The horizontal dashed line shows the zero level for this event. The time axis is logarithmic (after Strohmayer \& Brown 2002; Strohmayer \& Markwardt 2002).

giving decades long recurrence times and most of the energy released $\left(10^{43}-10^{44} \mathrm{ergs}\right)$ escapes as neutrinos or is conducted into the star, leaving $\approx 10^{42}$ ergs to emerge from the surface within a few hours (Strohmayer \& Brown 2002). Shorter recurrence times are possible if a smaller mass of carbon can be triggered somehow. Large carbon fractions in the ocean are expected for $4 \mathrm{U}$ 1820-30, since stable helium burning at the higher inferred mass accretion rates, when normal (10 - 20 second duration) bursts are not observed, will produce lots of carbon.

Pure carbon is unlikely to apply to the superbursts from $\mathrm{H} / \mathrm{He}$ accretors as Schatz et al. $(1999,2001)$ have shown that only a small amount of carbon remains after the burning of $\mathrm{H}$ and $\mathrm{He}$ via the rp-process. However, Cumming \& Bildsten (2001) (hereafter CB01) showed that even small amounts of carbon can be a promising energy source for the superbursts. They found that burning of this small mass fraction of carbon is thermally unstable at low accumulated masses when the ocean contains heavy ashes from the rp-process.

CB01 proved the important role played by the rp-process ashes. Their low thermal 


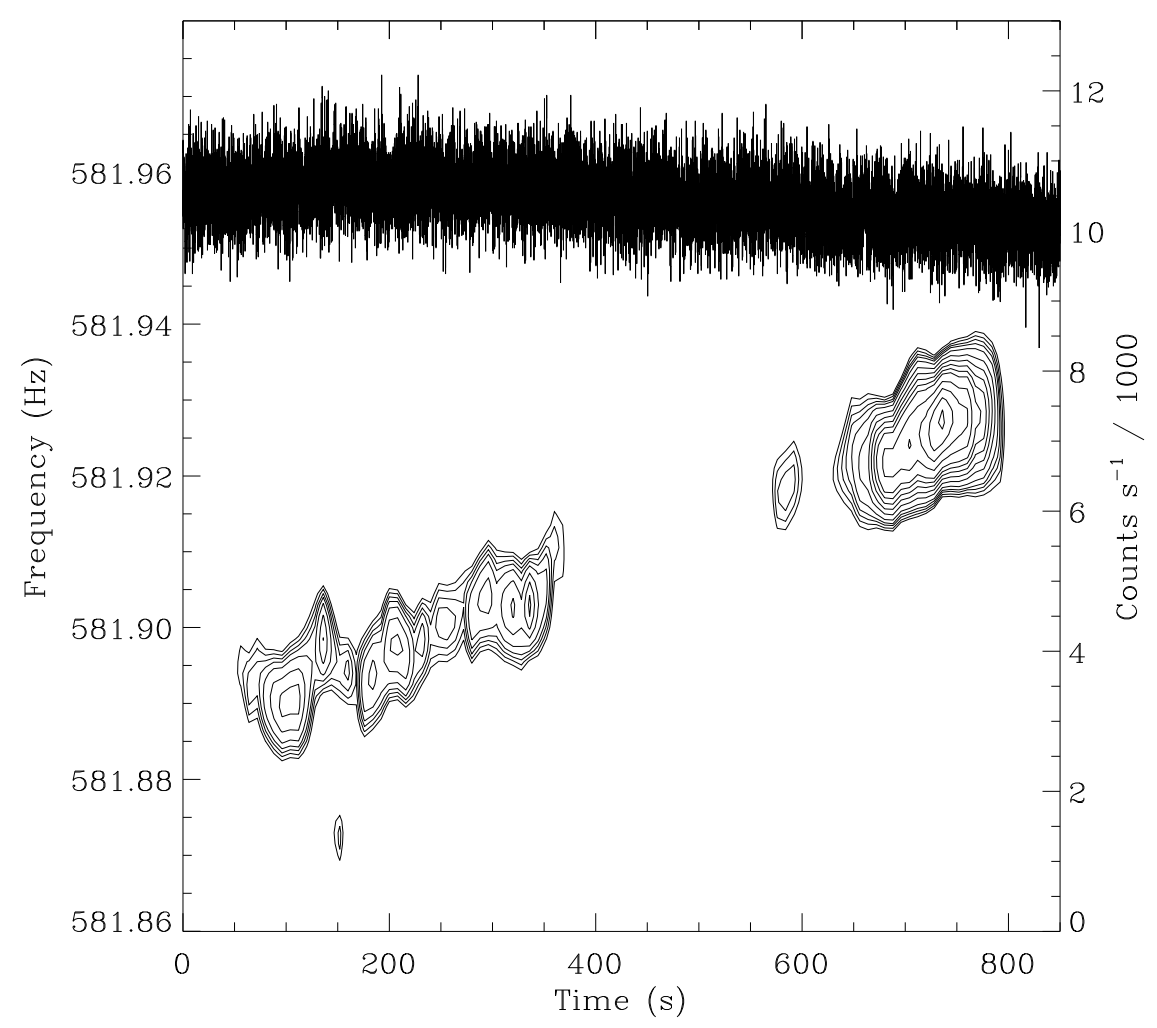

Fig. 3.16. Dynamic power spectrum of a portion of the February 22, 2001 superburst from $4 \mathrm{U} 1636-53$ showing $582 \mathrm{~Hz}$ pulsations. Shown are contours of constant $Z_{1}^{2}$ power versus frequency (left axis), and the RXTE/PCA countrate (right axis), both as a function of time. The monotonic increase in the pulsation frequency is consistent with binary orbital modulation (after Strohmayer \& Markwardt 2002).

conductivity gives a large temperature gradient in the ocean, so that the trace carbon ignites at accumulated masses comparable to that observed. The resulting energies, recurrence times, and conductive cooling times can easily accommodate the observed properties of superbursts (see Figure 3.17), especially now that an extra energy source was found by Schatz, Bildsten \& Cumming (2003). They showed that the conversion of rp-process nuclei back to the iron group elements during the carbon-triggered flash can enhance the energy release from that given in CB01 by factors of four.

The instability requires that $\dot{M}>0.1 \dot{M}_{\text {Edd }}$ when the carbon mass fraction is less than $10 \%$. Lower $\dot{M}$ 's stably burn the carbon. Though the instability is present at accretion rates $\approx \dot{M}_{\mathrm{Edd}}$, those flashes provide less of a contrast with the accretion luminosity, thus explaining why detection is easier when $\dot{M} \approx(0.1-0.3) \dot{M}_{\text {Edd }}$. Detecting one of these flares from a rapidly accreting $\mathrm{Z}$ source requires flux sensitivity at the $10 \%$ level on a timescale of a few hours and spectral sensitivity to distinguish that the flux rise is from extra thermal emission. This should be carried out and would confirm the notion of trace carbon ignition in the heavy rp-process ashes.

The energy from these mixed flashes takes a long time to escape the star, possibly 


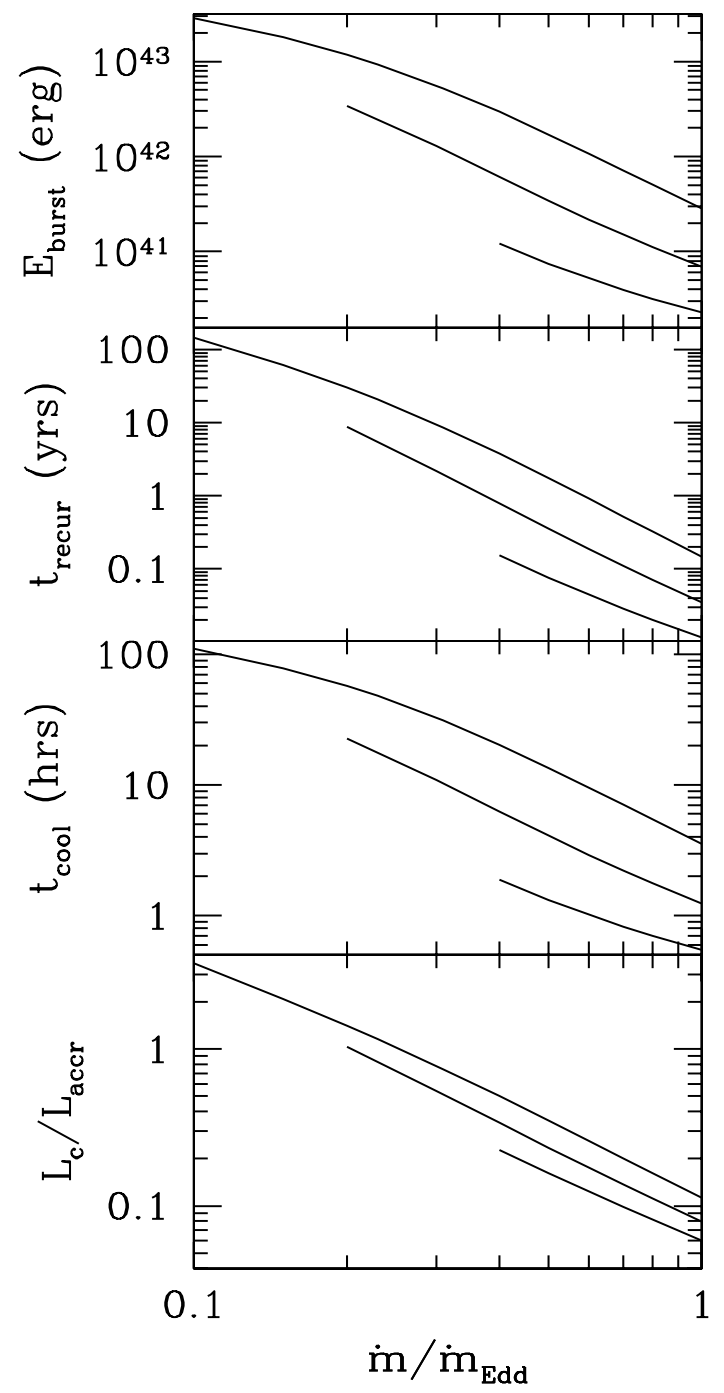

Fig. 3.17. Results of theoretical calculations of carbon flashes in the heavy element ocean on a neutron star. Shown from top to bottom are the burst energies, recurrence times, luminosity decay times, and the ratio of cooling luminosity to accretion luminosity, as a function of $\dot{m}$. The curves begin at the lowest accretion rate at which the thermal instability occurs in the heavy element $\left({ }^{104} \mathrm{Ru}\right)$ ocean. (after Cumming \& Bildsten 2001).

explaining the persistent "offset" in the flux nearly a day after the superburst in Ser X-1 (see Figure 3.14). CB01 also attributed the halting of regular Type I bursts after the superbursts to thermal stabilization of the $\mathrm{H} / \mathrm{He}$ burning layers by the large heat flux from the cooling ashes of the carbon burning. Paczynski (1983) and Bildsten (1995) have shown that luminosities in excess of the helium burning flux (or $L>L_{\text {accr }} / 100$ ) will stabilize the $\mathrm{H} / \mathrm{He}$ burning. This thus provides additional evidence that the superbursts are from burning deep within the neutron star. 
An alternative to carbon scenarios has been discussed by Kuulkers et al. (2002c). They suggest that electron captures on protons might be able to supply sufficient energy to power superbursts. The Fermi energy at column depths required to account for superburst energetics is close to the proton - neutron mass difference, so that protons can easily capture electrons. The resulting neutrons can then be captured on heavy nuclei, supplying about $7 \mathrm{MeV}$ per nucleon (Bildsten \& Cumming 1998). It is not yet clear whether this process is thermally unstable under the relevant conditions, and it is not certain that sufficient hydrogen can survive the initial hydrogen/helium burning. Further theoretical work should be able to answer these questions.

The initial theoretical understanding of superbursts is an exciting development as it further motivates the nucleosynthesis studies during Type I bursts and connects the ashes from the rp-process burning to the superburst explosions. We also hope that future observations will find the equivalent of superbursts from the higher accretion rate $\mathrm{Z}$ sources, or even from accreting X-ray pulsars.

\subsection{Summary and Future Prospects}

There has clearly been tremendous growth in our observational understanding of thermonuclear bursts in the past decade. Indeed, we think it is fair to say that the observations have gotten significantly ahead of the theory at the time of this writing. Yet, the promise of probing neutron star structure, and the fundamental physics needed to describe it, with burst observations has never been greater. Our understanding, though still incomplete, of the new phenomena, as well as insights drawn from previous observations, are sufficient to show that researchers must really begin to explore fully three dimensional and time dependent calculations of nuclear energy production and radiation transport in the neutron star surface layers in order to fully exploit the new phenomena as probes of neutron star structure and fundamental physics. The days of spherically symmetric, static models being adequate are long gone in our opinion.

In the context of burst oscillations it seems clear that the spreading of the nuclear burning front has important observational consequences, particularly for oscillations seen near the onset of bursts. A thorough theoretical understanding will only come with multi-dimensional dynamical studies of nuclear ignition and propagation. Indeed, higher signal to noise observations of the oscillations during burst rise could in principle provide us with a snapshot view of how burning spreads. Such studies will require even larger X-ray collecting areas than RXTE. A future goal would be to have detailed theoretical models of front propagation available for comparison with new data by the time that such observations are eventually made. Moreover, the persistence of oscillations in the cooling phase suggests that the dynamics of burst heating and rapid rotation combine to produce quasi-stable patterns in the surface layers that can persist for thousands of rotation periods (Spitkovsky et al. 2002). It is a triumph of modern X-ray astronomy that we are able to infer changes in the surface layers on neutron stars on the scale of only 10's of meters at distances of kiloparsecs! Helioseismology has revolutionized study of the solar interior, perhaps future improvements in sensitivity will allow a similar revolution in neutron star studies by enabling the detection of global oscillation modes of neutron stars.

As we have shown, an understanding of superbursts likely requires knowledge of 
the by-products of the nuclear burning. Detailed models of the heat and radiation transport from the deep ocean to the surface will be required to accurately model surface fluxes and time profiles and should be able to inform us about the location and conditions where superbursts are triggered. The existing RXTE observations with high signal to noise but low spectral resolution have shown that superbursts are likely promising targets for higher resolution spectroscopy, one of the goals of which should be to detect line features from neutron star surfaces. If such observations are to be possible, then X-ray monitoring instruments must be in place which can generate the necessary triggers. The discovery of superbursts was made possible with intensive all sky X-ray monitoring. Future efforts to study superbursts, for example, to better constrain the recurrence times, will require continued and improved X-ray monitoring capabilities. Future missions which should be able to address these needs include, INTEGRAL, Swift, Lobster-ISS, and MAXI.

High spectral resolution studies of bursts have begun with the new capabilities of Chandra and XMM/Newton. Frustratingly, the present data suggests that isolated neutron stars may be poor targets for line searches perhaps because of a dearth of metals in the atmosphere. Accreting neutron stars could be more tempting targets, with a continuous supply of metals furnished by the mass donor. Although the new generation of X-ray observatories have impressive capabilities, they still lack the collecting area necessary to obtain high signal to noise spectra from single bursts. To do better will require larger area missions, such as NASA's Constellation-X and ESA's XEUS.

We thank Erik Kuulkers, Craig Markwardt, Deepto Chakrabarty, Hendrik Schatz and Jean Swank for comments on the manuscript. We are indebted to Mike Muno, Andrew Cumming, Jean Cottam, Lucia Franco, Hendrik Schatz, Duncan Galloway, Remon Cornelisse, Erik Kuulkers and Anatoly Spitkovsky for either providing graphics or allowing us the use of previously published figures. We sincerely thank them all. We would like to dedicate this work to the memory of John C. L. Wang, a friend and colleague who left us much too soon. This work was supported by the National Science Foundation under grants PHY99-07949 and AST02-05956, NASA through grant NAG 5-8658. L. B. is a Cottrell Scholar of the Research Corporation.

\section{References}

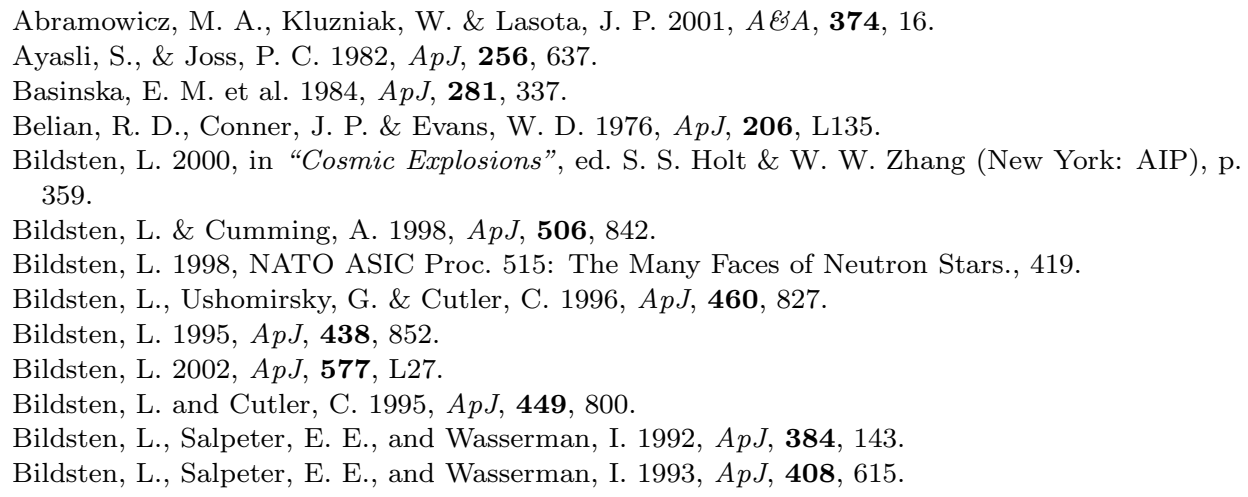


Bildsten, L. and Brown, E. F. 1997, ApJ, 477, 897.

Boirin, L., et al. 2000, $A \mathscr{E} A, \mathbf{3 6 1}, 121$

Braje, T. M., Romani, R. W., \& Rauch, K. P. 2000, ApJ, 531, 447.

Brown, E. F. \& Bildsten, L. 1998, ApJ, 496, 915.

Brown, E. F., Bildsten, L. \& Rutledge, R. E. 1998, ApJ, 504, L95.

Brown, E. F. 2000, ApJ, 531, 988

Burwitz, V., Zavlin, V. E., Neuhauser, R., Predehl, P., Trumper, J., \& Brinkman, A. C. 2001, $A \& A, \mathbf{3 7 9}, \mathrm{L} 35$

Chakrabarty, D. 2002, American Physical Society, Meeting ID: APR02, abstract \#S11.003, 11003.

Chakrabarty, D. 2000, Talk presented at AAS HEAD meeting, Honolulu, HI

Chakrabarty, D. \& Morgan, E. H. 1998, Nature, 394, 346.

Chen, K. \& Shaham, J. 1989, ApJ, 339, 279.

Chevalier, C. \& Ilovaisky, S. A. 1990, A\&A, 228, 119.

Cocchi, M. et al. 2001, $A \mathscr{E} A, \mathbf{3 7 8}$, L37.

Cocchi, M. et al. 2000, in "Proceedings of the Fifth Compton Symposium", ed. M. L. McConnell \& J. M. Ryan (New York:AIP), p. 203.

Colpi, M., Geppert, U., Page, D., Possenti, A. 2001, ApJ, 548, L175

Cornelisse, R. et al. 2002a, $A \notin \mathcal{E} A, \mathbf{3 9 2}, 885$.

Cornelisse, R., Verbunt, F., in 't Zand, J. J. M., Kuulker, E. \& Heise, J. 2002b, A\&A, 392, 931.

Cornelisse, R., Kuulkers, E., in't Zand, J. J. M., Verbunt, F., \& Heise, J. 2002c, AESA, 382, 174.

Cornelisse, R., Heise, J., Kuulkers, E., Verbunt, F., \& in't Zand, J. J. M. 2000, A\&A, 357, L21.

Cottam, J., Paerels, F. \& Mendez, M. 2002, Nature, 420, 51.

Cottam, J., Kahn, S. M., Brinkman, A. C., den Herder, J. W., \& Erd, C. 2001, A $\& A$, 365, L277

Cumming, A., Morsink, S. M., Bildsten, L., Friedman, J. L., \& Holz, D. E. 2002, ApJ, 564, 343.

Cumming, A., Zweibel, E. \& Bildsten, L. 2001, ApJ, 557, 958.

Cumming, A. \& Bildsten, L. 2001, ApJ, 559, L127.

Cumming, A. \& Bildsten, L. 2000, ApJ, 544, 453.

Damen, E. et al. 1990, $A \& \& A, \mathbf{2 3 7}, 103$.

Day, C. S. R., Fabian, A. C. \& Ross, R. R. 1992, MNRAS, 257, 471.

Day, C. S. R. \& Done, C. 1991, MNRAS, 253, 35P.

Drake, J. J. et al. 2002, ApJ, 572, 996.

Ebisuzaki, T. \& Nakamura, N. 1988, ApJ, 328, 251.

Ebisuzaki, T. 1987, Publ. Astron. Soc. Japan, 39, 287.

Ford, E. C. 2000, ApJ, 535, L119.

Ford, E. C. 1999, ApJ, 519, L73.

Foster, A. J., Fabian, A. C., \& Ross, R. R. 1987, MNRAS, 228, 259.

Fowler, W. A. \& Hoyle, F. 1965, Nucleosynthesis in massive stars and supernovae, Chicago: University of Chicago Press.

Fox, D. W., Muno, M. P., Lewin, W. H. G., Morgan, E. H., \& Bildsten, L. 2001, American Astronomical Society Meeting, 198

Franco, L. 2000, ApJ, 554, 340.

Franco, L. M. \& Strohmayer, T. E. 1999, BAAS, 31, 1556.

Fryxell, B. A., \& Woosley, S. E. 1982, ApJ, 261, 332.

Fujimoto, M. Y., Sztajno, M., Lewin, W. H. G. \& van Paradijs, J. 1987, ApJ, 319, 902.

Fujimoto, M. Y. \& Taam, R. E. 1986, ApJ, 305, 246.

Fujimoto, M. Y., Hanawa, T., \& Miyaji, S. 1981, ApJ, 247, 267.

Fushiki, I. \& Lamb, D. Q. 1987, ApJ, 323, L55.

Galloway, D. K., Chakrabarty, D., Morgan, E. H. \& Remillard, R. A. 2002, ApJ, 576, L137.

Galloway, D. K., Chakrabarty, D., Muno, M. P., \& Savov, P. 2001, ApJ, 549, L85.

Galloway, D. K., Kuulkers, E., Bildsten, L. \& Chakrabarty, D. 2003, submitted to ApJ

Giles, A. B., Hill, K. M., Strohmayer, T. E. \& Cummings, N. 2002, ApJ, 568, 279.

Giles, A. B. et al. 1996, ApJ, 469, L25.

Gottwald, M. et al. 1989, $A p J, \mathbf{3 3 9}, 1044$.

Grindlay, J. E. et al. 1976, ApJ, 205, L127.

Haberl, F. \& Titarchuk, L. 1995, A\&A A, 299, 414.

Haensel, P. \& Zdunik, J. L. 1990, A\&A, 227, 431

Hanawa, T. \& Sugimoto, D. 1988, Publ. Astron. Soc. Japan, 34, 1. 
Hanawa, T. \& Fujimoto, M. Y. 1984, Publ. Astron. Soc. Japan, 36, 119.

Hanawa, T., Sugimoto, D. \& Hashimoto, M. 1983, Publ. Astron. Soc. Japan, 35, 491.

Hansen, C. J. \& van Horn, H. M. 1975, ApJ, 195, 735.

Hasinger, G. \& van der Klis, M. 1989, $A \& A$, 225, 79.

Heyl, J. S., 2000, ApJ, 542, L45.

Hoffman, J. A., Lewin, W. H. G. \& Doty, J. 1977, ApJ, 217, L23

Inogamov, N. A. \& Sunyaev, R. A. 1999, Astron. Letters., 25, 269.

in 't Zand, J. J. M. et al. 2002, $A \& B A, \mathbf{3 8 9}, \mathrm{L} 43$.

in 't Zand, J. J. M. et al. 2001, $A \mathscr{E} A, \mathbf{3 7 2}, 916$.

in 't Zand, J. J. M. 2001, in Exploring the gamma-ray universe, ed. A. Gimenez, V. Reglero, \& C. Winkler, ESA Pub. Div., p. 463.

in 't Zand, J.J.M., Heise, J., Kuulkers, E., Bazzano, A., Cocchi, M., \& Ubertini, P. 1999, A\& A, 347, 891.

Jager, R., Mels, W. A., Brinkman, A. C., et al. 1997, $A \& A S$, 125, 557.

Jongert, H. C. \& van der Klis, M. 1996, $A \& A$, 310, 474.

Jonker, P. G., Mendez, M. \& van der Klis, M. 2002, MNRAS, 336, L1.

Joss, P. C. 1977, Nature, 270, 310.

Joss, P. C. 1978, ApJ, 225, L123.

Joss, P. C. \& Li, F. L. 1980, ApJ, 238, 287

Joss, P. C. \& Melia, F. 1987, ApJ, 312, 700

Kaminker, A. D. et al. 1990, Astrophys. Space Sci., 173, 171.

Kaptein, R. G. et al. 2000, $A \xi \exists A$, 358, L71.

Kluzniak, W. \& Wagoner, R. V. 1985, ApJ, 297, 548.

Kluzniak, W., Michelson, P. \& Wagoner, R. V. 1990, ApJ, 358, 538.

Kluzniak, W. \& Wilson, J. R. 1991, ApJ, 372, 87.

Koike, O., Hashimoto, M., Arai, K., Wanajo, S. 1999, A\&AA, 342, 464.

Kong, A. K. H. et al. 2000, MNRAS, 311, 405.

Kouveliotou, C. et al. 1996, Nature, 379, 799.

Kuulkers, E., den Hartog, P. R., in 't Zand, J. J. M., Verbunt, F. W. M., Harris, W. E. \& Cocchi, M. 2002a, $A \mathscr{E} A$, in press.

Kuulkers, E., Homan, J., van der Klis, M., Lewin, W. H. G. \& Mendez, M. 2002b, A\&A, 382, 947.

Kuulkers, E. et al. 2002c, $A \& A, \mathbf{3 8 2}, 503$.

Kuulkers, E. 2002, A\&SA, 383, L5.

Kuulkers, E. \& van der Klis, M. 2000, $A \& 3 A$, 356, L45.

Lamb, D. Q. \& Lamb, F. K. 1978, ApJ, 220, $291 \mathrm{~L}$.

Lamb, F. K. \& Miller, M. C. 2001, ApJ, 554, 1210.

Langmeier, A. et al. 1987, ApJ, 323, 288.

Lapidus, I., Nobili, L. \& Turolla, R. 1994, ApJ, 431, L103.

Lattimer, J. M. \& Prakash, M. 2001, ApJ, 550, 426

Levine, A. M. et al. 1996, ApJ, 469, L33.

Lewin, W. H. G., van Paradijs, J. \& Taam, R. E. 1993, Space Sci. Rev., 62, 223.

Lewin, W. H. G., Vacca, W. D. \& Basinska, E. M. 1984, ApJ, 277, L57.

Lewin, W. H. G. 1982, in Accreting Neutron Stars, W. Brinkman \& J. Trümper (eds.), MPE

Report 177, ISSN 0340-8922, 176

Liu, Q. Z., van Paradijs, J. \& van den Heuvel, E. P. J. 2001, A\&A, 368, 1021.

Livio, M. \& Bath, G. T. 1982, A\&A,116, 286.

London, R. A., Howard, W. M. \& Taam, R. E. 1984, ApJ, 287, L27.

London, R. A., Howard, W. M. \& Taam, R. E. 1986, ApJ, 306, 170.

Madej, J. 1991, ApJ, 376, 161

Magnier, E. et al. 1989, MNRAS, 237, 729.

Maraschi, L. \& Cavaliere, A. 1977, in Highlights in Astronomy, ed. E. A Müller, (Reidel, Dordrecht), Vol. 4, Part I, 127.

Markwardt, C. B., Miller, J. M. \& Wijnands, R. 2002, IAUC, 7993.

Markwardt, C. B., Strohmayer, T. E., \& Swank, J. H. 1999, ApJ, 512, L125.

Marshall, H. L. 1982, ApJ, 260, 815.

Mason, K. O., Middleditch, J., Nelson, J. E., \& White, N. E. 1980, Nature, 287, 516.

McDermott, P. N., and Taam, R. E. 1987, ApJ, 318, 278. 
Mendez, M. \& van der Klis, M. 1999, ApJ, 517, L51.

Mendez, M., van der Klis, M. \& van Paradijs, J. 1998, ApJ, 506, L117.

Mereghetti, S. et al. 2003, to appear in $A p J$

Miller, M. C. 2000, ApJ, 531, 458.

Miller, M. C. 1999, ApJ, 515, L77.

Miller, M. C. \& Lamb, F. K. 1998, ApJ, 499, L37.

Miller, M. C., Lamb, F. K. \& Psaltis, D. 1998, ApJ, 508, 791.

Muno, M. P., Özel, F. \& Chakrabarty, D. 2002, ApJ, in press.

Muno, M. P., Chakrabarty, D., Galloway, D. K. \& Psaltis, D. 2002, ApJ, in press.

Muno, M. P., Chakrabarty, D., Galloway, D. K., \& Savov, P. 2001, ApJ, 553, L157.

Muno, M. P., Fox, D. W., Morgan, E. H. \& Bildsten, L. 2000, ApJ, 542, 1016.

Murakami, T., Inoue, H., Makishima, K. \& Hoshi, R. 1987, Publ. Astron. Soc. Japan, 39, 879.

Nakamura, N., Inoue, H., \& Tanaka, Y. 1988, Publ. Astron. Soc. Japan, 40, 209.

Nath, N. R., Strohmayer, T. E., \& Swank, J. H. 2002, ApJ, 564, 353.

Nayakshin, S. \& Kallman, T. R. 2001, ApJ, 546, 406.

Nozakura, T., Ikeuchi, S. \& Fujimoto, M. Y. 1984, ApJ, 286, 221.

Paczynski, B. \& Anderson, N. 1986, ApJ, 302, 1.

Paczynski, B. 1983, ApJ, 264, 282.

Pavlov, G. G. et al. 2001, ApJ, 552, L129.

Pechenick, K. R., Ftaclas, C., \& Cohen, J. M. 1983, ApJ, 274, 846.

Pinto, P. A., Taam, R. E., \& Laming, J. M. 1991, BAAS, 23, 1321.

Rappaport, S., Joss, P. C. \& Webbink, R. F. 1982, ApJ, 254, 616.

Robinson, E. L. \& Young, P. 1997, ApJ, 491, L89.

Ross, R. R., Fabian, A. C. \& Young, A. J. 1999, MNRAS, 306, 461.

Rutledge, R. E., Bildsten, L., Brown, E. F., Pavlov, G. G. \& Zavlin, V. E. 2001, ApJ, 577, 405.

Sadeh, D. et al. 1982, ApJ, 257, 214.

Sanwal, D., Pavlov, G. G., Zavlin, V. E. \& Teter, M. A. ApJ, 574, L61.

Schatz, H. et al. 2001, Phys. Rev. Lett., 86, Number 16, 3471,

Schatz, H., Bildsten, L., Cumming, A. and Wiescher, M. 1999, ApJ, 524, 1014.

Schatz, H., Bildsten, L., Cumming, A. 2003, to appear in ApJ

Schatz, H. et al. 1998, Phys. Reports, 294, 167.

Schoelkopf, R. J. \& Kelley, R. L. 1991, ApJ, 375, 696.

Schwarzschild, M. \& Härm, R. 1965, ApJ, 142, 855.

Shaposhnikov, N. \& Titarchuk, L. 2002, ApJ, 567, 1077.

Shara, M. M. 1982, ApJ, 261, 649.

Smale, A. P. 2001, ApJ, 562, 957.

Smale, A. P. 1998, ApJ, 498, L141.

Smith, D., Morgan, E. H. \& Bradt, H. V. 1997, ApJ, 479, L137.

Spitkovsky, A., Levin, Y. \& Ushomirsky, G. 2002, ApJ, 566, 1018.

Strohmayer, T. E. \& Markwardt, C. B. 2002, ApJ, 577, 337.

Strohmayer, T. E. \& Brown, E. F. 2002, ApJ, 566, 1045.

Strohmayer, T. E. 2001, Adv. Space Res., 28, 511.

Strohmayer, T. E. 2000, AAS/High Energy Astrophysics Division, 32.

Strohmayer, T. E. \& Markwardt, C. B. 1999, ApJ, 516, L81.

Strohmayer, T. E. 1999, ApJ, 523, L51.

Strohmayer, T. E., Swank, J. H. \& Zhang, W. 1998, Nuclear Phys B., (Proceedings Supplement), 69/1-3, 129.

Strohmayer, T. E. et al. 1998a, ApJ, 498, L135.

Strohmayer, T. E. et al. 1998b, ApJ, 503, L147.

Strohmayer, T. E., Zhang, W. \& Swank, J. H. 1997, ApJ, 487, L77.

Strohmayer, T. E., Jahoda, K., Giles, A. B. \& Lee, U. 1997, ApJ, 486, 355.

Strohmayer, T. E. and Lee, U. 1996, $A p J, \mathbf{4 6 7}, 773$.

Strohmayer, T. E. et al. 1996, ApJ, 469, L9.

Swank, J. H. et al. 1977, ApJ, 212, L73.

Sztajno, M. et al. 1987, MNRAS, 226, 39.

Taam, R. E., Woosley, S. E., \& Lamb, D. Q. 1996, ApJ, 459, 271.

Taam, R. E. \& Picklum, R.E. 1978, ApJ, 224, 210. 
Titarchuk, L. \& Shaposhnikov, N. 2002, ApJ, 570, L25.

Titarchuk, L. 1994, ApJ, 429, 340.

Ubertini, P. et al. 1999, ApJ, 514, L27.

van der Klis, M. et al. 1990, $A p J$, 360, L19.

van Paradijs, J. et al. 1990, Publ. Astron. Soc. Japan, 42, 633.

van Paradijs, J., Penninx, W. \& Lewin, W. H. G. 1988, MNRAS, 233, 437.

van Paradijs, J. \& Lewin, W. H. G. 1987, A\&A, 172, L20.

van Paradijs, J. 1981, $A \& A$, 101, 174.

van Paradijs, J. 1978, Nature, 274, 650.

van Straaten, S. et al. 2000, ApJ, 551, 907.

Waki, I. et al. 1984, Publ. Astron. Soc. Japan, 36, 819.

Wallace, R. K., \& Woosley, S. E. 1984, in "High Energy Transients in Astrophysics", ed. S.E. Woosley (New York: AIP), p. 273.

Wallace, R. K., \& Woosley, S. E. 1981, ApJS 43, 389.

Wallace, R. K., Woosley, S. E. \& Weaver, T. A. 1982, ApJ 258, 696.

Walter, F. M. \& Lattimer, J. M. 2002, ApJ, 576, L145.

Weinberg, N., Miller, M. C., \& Lamb, D. Q. 2001, ApJ, 546, 1098.

Wijnands, R. et al. 2002, private communication.

Wijnands, R. et al. 2002, ApJ, 566, 1060.

Wijnands, R. 2001, ApJ, 554, L59.

Wijnands, R. Strohmayer, T. E. \& Franco, L. M. 2001, ApJ, 549, L71.

Wijnands, R. \& van der Klis, M. 1998, Nature, 394, 344.

Wijnands, R., \& van der Klis, M. 1997, ApJ, 482, L65.

Woosley, S. E. \& Taam, R. E. 1976, Nature, 263, 101.

Yu, W., Li, T. P., Zhang, W., \& Zhang, S. N. 1999, ApJ, 512, L35.

Zhang, W. et al. 1998, ApJ, 495, L9.

Zingale, M. et al. 2001, ApJS, 133, 195. 